\title{
Infinite-dimensional Lur'e systems with almost periodic forcing
}

\author{
Max E. Gilmore ${ }^{1} \cdot$ C. Guiver ${ }^{1}$ (D) H. Logemann ${ }^{1}$
}

Received: 24 September 2019 / Accepted: 27 July 2020 / Published online: 10 August 2020

(c) The Author(s) 2020

\begin{abstract}
We consider forced Lur'e systems in which the linear dynamic component is an infinitedimensional well-posed system. Numerous physically motivated delay and partial differential equations are known to belong to this class of infinite-dimensional systems. We present refinements of recent incremental input-to-state stability results (Guiver in SIAM J Control Optim 57:334-365, 2019) and use them to derive convergence results for trajectories generated by Stepanov almost periodic inputs. In particular, we show that the incremental stability conditions guarantee that for every Stepanov almost periodic input there exists a unique pair of state and output signals which are almost periodic and Stepanov almost periodic, respectively. The almost periods of the state and output signals are shown to be closely related to the almost periods of the input, and a natural module containment result is established. All state and output signals generated by the same Stepanov almost periodic input approach the almost periodic state and the Stepanov almost periodic output in a suitable sense, respectively, as time goes to infinity. The sufficient conditions guaranteeing incremental input-to-state stability and the existence of almost periodic state and Stepanov almost periodic output signals are reminiscent of the conditions featuring in well-known absolute stability criteria such as the complex Aizerman conjecture and the circle criterion.
\end{abstract}

Keywords Absolute stability - Almost periodic functions · Circle criterion . Incremental input-to-state stability · Infinite-dimensional systems · Lur'e systems . Small gain

Published in the topical collection Input-to-state stability for infinite-dimensional systems.

C. Guiver

c.guiver@bath.ac.uk

Max E. Gilmore

m.e.gilmore@bath.ac.uk

H. Logemann

h.logemann@bath.ac.uk

1 Department of Mathematical Sciences, University of Bath, Bath BA2 7AY, UK 
Mathematics Subject Classification 34C27 - 93C10 - 93C20 - 93C23 - 93C25 .

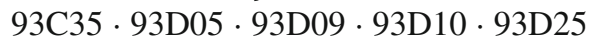

\section{Introduction}

The analysis of solutions of differential equations, the right-hand side of which exhibits almost periodic time dependence, has a long history and the relevant literature is vast, see, for example, $[1,8,9,14]$. Typical questions arising in this context are:

- Does there exist a unique almost periodic solution, and if so, are all solutions asymptotically almost periodic with long-term behaviour (in forward time) asymptotically identical to that of the unique almost periodic solution?

- Are bounded bilateral solutions almost periodic?

Whilst the current paper continues this tradition, we use input-to-state stability ideas from control theory which, to the best of our knowledge, have not been employed in this context before.

More specifically, we analyse the asymptotic behaviour of a large class of infinitedimensional Lur'e systems with Stepanov almost periodic inputs. We remark that the concept of almost periodicity in the sense of Stepanov generalizes that of Bohr, which, in the following, will be simply referred to as almost periodicity. Adopting the set-up considered in [16], we study the forced Lur'e system shown in Fig. 1, where $\Sigma$ is a well-posed ${ }^{1}$ linear infinite-dimensional system and $f$ is a static nonlinearity. Note that, in Fig. 1, the signals $v, y$ and $u$ are given by

$$
v=\left(\begin{array}{l}
v^{1} \\
v^{2}
\end{array}\right), \quad y=\left(\begin{array}{l}
y^{1} \\
y^{2}
\end{array}\right), \quad u=\left(\begin{array}{c}
v^{1} \\
f\left(y^{2}\right)+v^{2}
\end{array}\right) .
$$

We note that well-posed linear systems allow for considerable unboundedness of the control and observation operators and they encompass many of the most commonly studied partial differential equations with boundary control and observation, and a large class of functional differential equations of retarded and neutral type with delays in the inputs and outputs. There exists a highly developed state-space and frequencydomain theory for well-posed infinite-dimensional systems; see, for example, [29,30, $39,40,42,43,45-47]$.

Lur'e systems are a common and important class of nonlinear control systems, and the study of their stability properties is known as absolute stability theory (see, for example, [18-20,44,49]). Classical absolute stability theory comes in two flavours: in a state-space setting, unforced $(v=0)$ finite-dimensional systems are considered and the emphasis is on global asymptotic stability, whilst the input-output approach (initiated by Sandberg and Zames in the 1960s) focusses on $L^{2}$-stability and, to a lesser extent, on $L^{\infty}$-stability, see $[13,44]$. A more recent development is the analysis of state-space systems of Lur'e format in an input-to-state stability (ISS) context,

1 Throughout the paper, "well-posedness" refers to well-posedness in the $L^{2}$ sense, which is the natural setting, as frequency-domain methods, familiar from classical absolute stability theory, generalize nicely in this infinite-dimensional framework. 
Fig. 1 Block diagram of forced Lur'e system: the feedback interconnection of the well-posed linear system $\Sigma$ and the static nonlinearity $f$

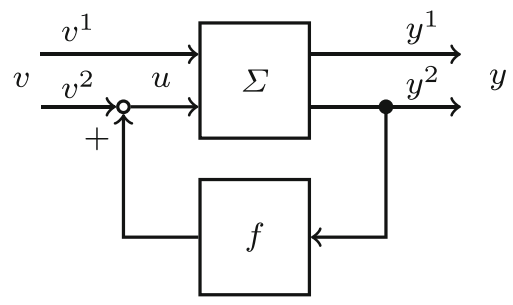

thereby, in a sense, merging the two strands of the earlier theory [3,16,21,22,35]. The ISS concept was introduced (for general nonlinear control systems) in [37] and further developed across a huge range of papers, see, for example, the survey articles [12,38]. ISS for infinite-dimensional control systems is an active area of research, see, for example, the research monograph [23] and the recent survey [25]. Lur'e systems often arise in a control-theoretic setting as the feedback interconnection of a linear system with a static nonlinear output feedback, such as integral control in the presence of input saturation, see $[10,17]$ for instance. However, we also note that certain nonlinear evolution equations admit a Lur'e structure, separate from any control-theoretic interpretation, see [36].

So far, the ISS approach to Lur'e systems is very much restricted to finitedimensional systems with [16] being one of the very few exceptions. In fact, in [16] a number of incremental ISS results are derived (the underlying concept inspired by that introduced in [2]) and are then applied to obtain convergence properties including the converging input-converging output property and the asymptotic periodicity of the state and output trajectories under periodic forcing. In this paper, we provide a refinement of the incremental ISS results in [16] and use them to analyse the asymptotic behaviour of the Lur'e system shown in Fig. 1 in response to Stepanov almost periodic inputs.

With regard to stability properties, our main result is Theorem 3.4, which is reminiscent of the complex Aizerman conjecture [19,20] (familiar from finite-dimensional control theory) and constitutes a refinement of [16, Theorem 4.1]. The main novelty here is that we obtain an incremental ISS estimate which is in terms of the Stepanov norm

$$
\|\Delta v\|_{S^{q}}:=\sup _{a \geq 0}\left(\int_{a}^{a+1}\|(\Delta v)(t)\|^{q} d t\right)^{1 / q}, \quad 2 \leq q<\infty
$$

where $\Delta v$ denotes the difference of two inputs. Our main concern in this paper is to analyse the behaviour of Lur'e systems subject to Stepanov almost periodic forcing. Based on the incremental ISS result in Theorem 3.4, we show that incremental versions of certain classical sufficient conditions for absolute stability such as the complex Aizerman conjecture [19,20], the small-gain theorem [13,44] and the circle criterion [22,44] (or variations thereof) guarantee that, for a given Stepanov almost periodic input $v^{*}$, there exists a corresponding unique state/output trajectory $\left(x^{*}, y^{*}\right)$ with $x^{*}$ almost periodic and $y^{*}$ Stepanov almost periodic, and, furthermore, for any input/state/output trajectory $(v, x, y)$ such that $v(t)$ approaches $v^{*}(t)$ as $t \rightarrow \infty$ in a 
natural sense, the behaviour of $(x, y)$ is asymptotically identical to that of $\left(x^{*}, y^{*}\right)$. The almost periods of $x^{*}$ and $y^{*}$ are shown to be closely related to the almost periods of $v^{*}$ in the sense that for every $\varepsilon>0$ there exists a $\delta>0$ such that every $\delta$-almost period of $v^{*}$ is an $\varepsilon$-almost period of $x^{*}$ and $y^{*}$. Furthermore, it is established that the modules generated by the frequency spectra of $x^{*}$ and $y^{*}$ are contained in that generated by the frequency spectrum of $v^{*}$. Our main results, in Theorems 4.5 and 4.7, provide far-reaching generalizations of earlier contributions in $[6,16,31-34,48]$. We discuss how our results relate to others in the literature at the end of Sect. 4.

The paper is organized as follows. Section 2 gathers notation and required material from the theory of well-posed linear systems. In Sect. 3, we introduce the Lur'e system shown in Fig. 1 in a formal way and then develop the key tool for our analysis of almost periodically forced Lur'e systems, namely a suitably refined version of the incremental ISS result [16, Theorem 4.1]. The main topic of the paper is addressed in Sect. 4: after a discussion of relevant background material from the theory of almost periodic functions (in the sense of Bohr and its generalization by Stepanov), we state and prove Theorems 4.5 and 4.7, the main results of this work. An example is presented in Sects. 5, and 6 contains some conclusions.

\section{Preliminaries}

Let $\mathbb{Z}$ be the set of integers and set

$$
\mathbb{Z}_{+}:=\{n \in \mathbb{Z}: n \geq 0\} \text { and } \mathbb{N}:=\{n \in \mathbb{Z}: n \geq 1\}
$$

For real or complex Hilbert spaces $U$ and $Y$, let $\mathcal{L}(U, Y)$ denote the space of all linear bounded operators mapping $U$ to $Y$. As usual, we set $\mathcal{L}(U):=\mathcal{L}(U, U)$. For $Z \in \mathcal{L}(U, Y)$ and $r>0$, define

$$
\mathbb{B}(Z, r):=\{T \in \mathcal{L}(U, Y):\|T-Z\|<r\},
$$

the open ball in $\mathcal{L}(U, Y)$, with centre $Z$ and radius $r$.

For $\alpha \in \mathbb{R}$, set $\mathbb{C}_{\alpha}:=\{s \in \mathbb{C}: \operatorname{Re} s>\alpha\}$. The space of all holomorphic and bounded functions $\mathbb{C}_{\alpha} \rightarrow \mathcal{L}(U, Y)$ is denoted by $H_{\alpha}^{\infty}(\mathcal{L}(U, Y))$. Endowed with the norm

$$
\|\mathbf{H}\|_{H_{\alpha}^{\infty}}:=\sup _{s \in \mathbb{C}_{\alpha}}\|\mathbf{H}(s)\|,
$$

$H_{\alpha}^{\infty}(\mathcal{L}(U, Y))$ is a Banach space. We write $H^{\infty}(\mathcal{L}(U, Y))$ for $H_{0}^{\infty}(\mathcal{L}(U, Y))$.

For an arbitrary Banach space $W$ and $t \geq 0$, define the projection operator $\mathbf{P}_{t}$ : $L_{\text {loc }}^{2}\left(\mathbb{R}_{+}, W\right) \rightarrow L^{2}\left(\mathbb{R}_{+}, W\right)$ by

$$
\left(\mathbf{P}_{t} w\right)(\tau)= \begin{cases}w(\tau), & \forall \tau \in[0, t] \\ 0, & \forall \tau>t\end{cases}
$$


For $\alpha \in \mathbb{R}, 1 \leq q \leq \infty$ and an interval $J \subset \mathbb{R}_{+}$, we define the weighted $L^{q}$-space

$$
L_{\alpha}^{q}(J, W):=\left\{w \in L_{\mathrm{loc}}^{q}(J, W): \exp _{\alpha} w \in L^{q}(J, W)\right\},
$$

where $\exp _{\alpha}: \mathbb{R} \rightarrow \mathbb{R}$ is given by $\exp _{\alpha}(t):=e^{\alpha t}$. Endowed with the norm

$$
\|w\|_{L_{\alpha}^{q}(J)}=\left\|\exp _{\alpha} w\right\|_{L^{q}(J)}
$$

$L_{\alpha}^{q}(J, W)$ is a Banach space. To simplify notation, we will write $\|w\|_{L_{\alpha}^{q}}$ for $\|w\|_{L_{\alpha}^{q}\left(\mathbb{R}_{+}\right)}$. In the following, let $R=\mathbb{R}_{+}$or $\mathbb{R}$. For $\tau \in R$, the shift operator $\mathbf{S}_{\tau}: L_{\text {loc }}^{1}(R, W) \rightarrow$ $L_{\text {loc }}^{1}(R, W)$ is given by $\left(\mathbf{S}_{\tau} w\right)(t)=w(t+\tau)$ for all $t \in R$. For later purposes, we define $B C(R, W)$ and $B U C(R, W)$ as the spaces of all, respectively, bounded continuous and bounded uniformly continuous functions. Endowed with the supremum norm, $B C(R, W)$ and $B U C(R, W)$ are Banach spaces. Moreover, we define the space of uniformly locally $q$-integrable functions $U L_{\text {loc }}^{q}(R, W)$ by

$$
U L_{\mathrm{loc}}^{q}(R, W):=\left\{w \in L_{\mathrm{loc}}^{q}(R, W): \sup _{a \in R} \int_{a}^{a+1}\|w(t)\|^{q} d t<\infty\right\},
$$

where $1 \leq q<\infty$. It is straightforward to show that, with the Stepanov norm

$$
\|w\|_{S^{q}}:=\sup _{a \in R}\left(\int_{a}^{a+1}\|w(t)\|^{q} d t\right)^{1 / q}
$$

$U L_{\mathrm{loc}}^{q}(R, W)$ is a Banach space. Furthermore, for every $b>0$, the functional

$$
w \mapsto \sup _{a \in R}\left(\int_{a}^{a+b}\|w(t)\|^{q} d t\right)^{1 / q}
$$

is a norm on $U L_{\text {loc }}^{q}(R, W)$ and this norm is equivalent to $\|\cdot\|_{S^{q}}$. A routine application of Hölder's inequality shows that

$$
\begin{aligned}
& U L_{\mathrm{loc}}^{q}(R, W) \subset U L_{\mathrm{loc}}^{p}(R, W) \text { and } \\
& \|v\|_{S^{p}} \leq\|v\|_{S^{q}} \forall v \in U L_{\mathrm{loc}}^{q}(R, W), \quad \text { where } 1 \leq p \leq q<\infty,
\end{aligned}
$$

and so the space $U L_{\mathrm{loc}}^{q}(R, W)$ is continuously embedded in $U L_{\mathrm{loc}}^{p}(R, W)$ for $1 \leq$ $p \leq q<\infty$. Furthermore, for $\alpha \geq 0$, we define

$$
U_{\alpha} L_{\mathrm{loc}}^{q}(R, W):=\left\{w \in U L_{\mathrm{loc}}^{q}(R, W): \lim _{t \rightarrow \infty}\left\|\mathbf{S}_{t}\left(\exp _{\alpha} w\right)\right\|_{S^{q}}=0\right\} .
$$

It is clear that $L_{\alpha}^{q}\left(\mathbb{R}_{+}, W\right) \subset U_{\alpha} L_{\text {loc }}^{q}\left(\mathbb{R}_{+}, W\right)$, and, if $\alpha>0$, then $U_{\alpha} L_{\text {loc }}^{q}\left(\mathbb{R}_{+}, W\right) \subset$ $L_{\beta}^{q}\left(\mathbb{R}_{+}, W\right)$ for all $\beta \in(0, \alpha)$.

Below we will provide a brief review of some material from the theory of well-posed systems; for more details, we refer the reader to [39,42,43,45-47]. Throughout, we 
shall be considering a well-posed linear system $\Sigma=(\mathbb{T}, \Phi, \Psi, \mathbb{G})$ with state space $X$, input space $U$ and output space $Y$. Here $X, U$ and $Y$ are separable complex Hilbert spaces, $\mathbb{T}=\left(\mathbb{T}_{t}\right)_{t \geq 0}$ is a strongly continuous semigroup on $X, \Phi=\left(\Phi_{t}\right)_{t \geq 0}$ is a family of bounded linear operators from $L^{2}\left(\mathbb{R}_{+}, U\right)$ to $X$ (input-to-state maps), $\Psi=\left(\Psi_{t}\right)_{t \geq 0}$ is a family of bounded linear operators from $X$ to $L^{2}\left(\mathbb{R}_{+}, Y\right)$ (state-to-output maps) and $\mathbb{G}=\left(\mathbb{G}_{t}\right)_{t \geq 0}$ is a family of bounded linear operators from $L^{2}\left(\mathbb{R}_{+}, U\right)$ to $L^{2}\left(\mathbb{R}_{+}, Y\right)$ (input-to-output maps). In order for $\Sigma$ to qualify as a well-posed system, these families of operators need to satisfy certain natural conditions, see $[39,43,45,46]$. Particular consequences of these conditions are the following properties:

$$
\Phi_{t} \mathbf{P}_{t}=\Phi_{t}, \quad \mathbf{P}_{t} \Psi_{t+\tau}=\Psi_{t}, \quad \mathbf{P}_{t} \mathbb{G}_{t+\tau} \mathbf{P}_{t}=\mathbf{P}_{t} \mathbb{G}_{t+\tau}=\mathbb{G}_{t} \quad \forall t, \tau \geq 0
$$

It follows that $\Phi_{t}$ extends in a natural way to $L_{\text {loc }}^{2}\left(\mathbb{R}_{+}, U\right)$ and there exist operators $\Psi_{\infty}: X \rightarrow L_{\text {loc }}^{2}\left(\mathbb{R}_{+}, Y\right)$ and $\mathbb{G}_{\infty}: L_{\text {loc }}^{2}\left(\mathbb{R}_{+}, U\right) \rightarrow L_{\text {loc }}^{2}\left(\mathbb{R}_{+}, Y\right)$ such that

$$
\mathbf{P}_{t} \Psi_{\infty}=\Psi_{t}, \quad \mathbf{P}_{t} \mathbb{G}_{\infty}=\mathbb{G}_{t} \quad \forall t \geq 0 .
$$

The operator $\mathbb{G}_{\infty}$ is right-shift invariant (and hence causal) and is called the inputoutput operator of $\Sigma$. Given an initial state $x^{0}$ and an input $u \in L_{\text {loc }}^{2}\left(\mathbb{R}_{+}, U\right)$, the corresponding state and output trajectories $x$ and $y$ of $\Sigma$ are defined by

$$
\left.\begin{array}{l}
x(t)=\mathbb{T}_{t} x^{0}+\Phi_{t} \mathbf{P}_{t} u \\
\mathbf{P}_{t} y=\Psi_{t} x^{0}+\mathbb{G}_{t} \mathbf{P}_{t} u
\end{array}\right\} \quad \forall t \geq 0
$$

respectively.

Let $(A, B, C)$ denote the generating operators of $\Sigma$. The operator $A$ is the generator of the strongly continuous semigroup $\mathbb{T}=\left(\mathbb{T}_{t}\right)_{t \geq 0}$, and the operators $B \in \mathcal{L}\left(U, X_{-1}\right)$ and $C \in \mathcal{L}\left(X_{1}, Y\right)$ are the unique operators satisfying

$$
\Phi_{t} u=\int_{0}^{t} \mathbb{T}_{t-\tau} B u(\tau) \mathrm{d} \tau \quad \forall u \in L^{2}\left(\mathbb{R}_{+}, U\right), \forall t \geq 0
$$

and

$$
\left(\Psi_{\infty} x^{0}\right)(t)=C \mathbb{T}_{t} x^{0} \quad \forall x^{0} \in X_{1}, \forall t \geq 0,
$$

where the spaces $X_{1}$ and $X_{-1}$, respectively, are the usual interpolation and extrapolation spaces associated with $A$ and $X$.

The transfer function $\mathbf{G}$ of $\Sigma$ has the property that $\mathbf{G} \in H_{\alpha}^{\infty}(\mathcal{L}(U, Y))$ for every $\alpha>\omega(\mathbb{T})$, where $\omega(\mathbb{T})$ denotes the exponential growth constant of $\mathbb{T}$. The relationship between $\mathbf{G}$ and the operators $(A, B, C)$ is expressed by the formula

$$
\frac{1}{s-z}(\mathbf{G}(s)-\mathbf{G}(z))=-C(s I-A)^{-1}\left(z I-A_{-1}\right)^{-1} B \quad \forall s, z \in \mathbb{C}_{\omega(\mathbb{T})}, s \neq z,
$$


see [39, equation (4.6.9)], where $A_{-1} \in \mathcal{L}\left(X, X_{-1}\right)$ extends $A$ to $X$ and, considered as an unbounded operator on $X_{-1}$, generates a semigroup on $X_{-1}$ which extends $\mathbb{T}$ to $X_{-1}$. Furthermore, for $\beta \in \mathbb{R}$, the operator $\mathbb{G}_{\infty}$ is in $\mathcal{L}\left(L_{\beta}^{2}\left(\mathbb{R}_{+}, U\right), L_{\beta}^{2}\left(\mathbb{R}_{+}, Y\right)\right)$ if, and only if, $\mathbf{G} \in H_{-\beta}^{\infty}(\mathcal{L}(U, Y))$, in which case

$$
\left\|\mathbb{G}_{\infty}\right\|_{\beta}=\|\mathbf{G}\|_{H_{-\beta}^{\infty}},
$$

where $\|\cdot\|_{\beta}$ denotes the $L_{\beta}^{2}$-induced operator norm. We remark that $\beta<-\omega(\mathbb{T})$ is sufficient for $\mathbb{G}_{\infty}$ to be in $\mathcal{L}\left(L_{\beta}^{2}\left(\mathbb{R}_{+}, U\right), L_{\beta}^{2}\left(\mathbb{R}_{+}, Y\right)\right)$. We also record that, for every $\beta<-\omega(\mathbb{T})$, there exist positive constants $\varphi$ and $\psi$ such that

$$
\left\|e^{\beta t} \Phi_{t} u\right\| \leq \varphi\left\|\mathbf{P}_{t} u\right\|_{L_{\beta}^{2}} \forall u \in L_{\mathrm{loc}}^{2}\left(\mathbb{R}_{+}, U\right), \forall t \geq 0
$$

and

$$
\left\|\Psi_{\infty} x^{0}\right\|_{L_{\beta}^{2}} \leq \psi\left\|x^{0}\right\| \quad \forall x^{0} \in X
$$

The system (2.2) is said to be optimizable if, for every $x^{0} \in X$, there exists $u \in L^{2}\left(\mathbb{R}_{+}, U\right)$, such that $x \in L^{2}\left(\mathbb{R}_{+}, X\right)$. Furthermore, we say that (2.2) is estimatable if, the "dual" system is optimizable, that is, for every $z^{0} \in X$, there exists $v \in L^{2}\left(\mathbb{R}_{+}, Y\right)$ such that the function $t \mapsto \mathbb{T}_{t}^{*} z^{0}+\Psi_{t}^{*} v$ is in $L^{2}\left(\mathbb{R}_{+}, X\right)$. We note that, by [24], optimizability is equivalent to exponential stabilizability and estimatability is equivalent to exponential detectability (where exponential stabilizability and detectability are understood in the sense of [39]).

An operator $K \in \mathcal{L}(Y, U)$ is said to be an admissible feedback operator for $\Sigma$ (or for $\mathbf{G}$ ) if there exists $\alpha \in \mathbb{R}$ such that $I-\mathbf{G} K$ is invertible in $H_{\alpha}^{\infty}(\mathcal{L}(Y))$ and we set

$$
\mathbf{G}^{K}:=(I-\mathbf{G} K)^{-1} \mathbf{G} .
$$

If $K \in \mathcal{L}(Y, U)$ is an admissible feedback operator, then, for every $t \geq 0$, the operator $I-\mathbb{G}_{t} K$ is invertible in $\mathcal{L}\left(L^{2}\left(\mathbb{R}_{+}, Y\right)\right)$, and $I-\mathbb{G}_{\infty} K$ has a causal inverse $(I-$ $\left.\mathbb{G}_{\infty} K\right)^{-1}$ (mapping $L_{\text {loc }}^{2}\left(\mathbb{R}_{+}, Y\right)$ into itself). Furthermore, if $K \in \mathcal{L}(Y, U)$ is an admissible feedback operator for $\Sigma$, then there exists a unique well-posed system $\Sigma^{K}=\left(\mathbb{T}^{K}, \Phi^{K}, \Psi^{K}, \mathbb{G}^{K}\right)$ such that

$$
\Sigma_{t}^{K}=\Sigma_{t}+\Sigma_{t}\left(\begin{array}{cc}
0 & 0 \\
0 & K
\end{array}\right) \Sigma_{t}^{K} \quad \forall t \geq 0,
$$

where

$$
\Sigma_{t}:=\left(\begin{array}{ll}
\mathbb{T}_{t} & \Phi_{t} \\
\Psi_{t} & \mathbb{G}_{t}
\end{array}\right), \quad \Sigma_{t}^{K}:=\left(\begin{array}{ll}
\mathbb{T}_{t}^{K} & \Phi_{t}^{K} \\
\Psi_{t}^{K} & \mathbb{G}_{t}^{K}
\end{array}\right)
$$


Fig. 2 Block diagram of closed-loop feedback system of $\Sigma$ in connection with output feedback $K$

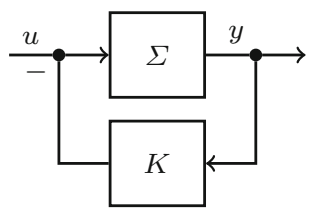

The transfer function of $\Sigma^{K}$ is $\mathbf{G}^{K}$. The interpretation of (2.3) is that $\Sigma^{K}$ is the closed-loop system shown in Fig. 2.

We say that an operator $K \in \mathcal{L}(Y, U)$ stabilizes $\mathbf{G}$ (or stabilizes $\Sigma$ in the inputoutput sense) if $\mathbf{G}^{K} \in H^{\infty}(\mathcal{L}(U, Y))$. The set of all operators stabilizing $\mathbf{G}$ is denoted by $\mathbb{S}(\mathbf{G})$. Trivially, every element in $\mathbb{S}(\mathbf{G})$ is an admissible feedback operator for $\mathbf{G}$.

The following lemma is a special case of [15, Proposition 5.6].

Lemma 2.1 For $K \in \mathcal{L}(Y, U)$ and $r>0, \mathbb{B}(K, r) \subset \mathbb{S}(\mathbf{G})$ if, and only if, $\left\|\mathbf{G}^{K}\right\|_{H^{\infty}} \leq$ $1 / r$.

In particular, if $K \in \mathbb{S}(\mathbf{G})$ and $\left\|\mathbf{G}^{K}\right\|_{H^{\infty}}>0$, then $\rho:=1 /\left\|\mathbf{G}^{K}\right\|_{H^{\infty}}$ is the largest number such that $\mathbb{B}(K, \rho) \subset \mathbb{S}(\mathbf{G})$.

An immediate consequence of the sufficiency part of Lemma 2.1 is that $\mathbb{S}(\mathbf{G})$ is an open subset of $\mathcal{L}(Y, U)$. Note that the sufficiency part is simply a version of the small-gain theorem. The assumption that the Hilbert spaces $U$ and $Y$ are complex plays an important role in the necessity part which in general does not hold for real Hilbert spaces.

In the following, we shall adopt the four-block setting for Lur'e systems considered in [16], see Fig. 1. In particular, we assume that the input and output spaces $U$ and $Y$ are of the form $U=U^{1} \times U^{2}$ and $Y=Y^{1} \times Y^{2}$, where $U^{i}$ and $Y^{i}$ are complex Hilbert spaces, $i=1$, 2. It is convenient to introduce the following maps

$$
P^{i}: Y \rightarrow Y^{i},\left(\begin{array}{l}
y^{1} \\
y^{2}
\end{array}\right) \mapsto y^{i}, \quad i=1,2,
$$

and

$$
E^{1}: U^{1} \rightarrow U, u \mapsto\left(\begin{array}{l}
u \\
0
\end{array}\right), \quad E^{2}: U^{2} \rightarrow U, u \mapsto\left(\begin{array}{l}
0 \\
u
\end{array}\right)
$$

If $y \in L_{\text {loc }}^{2}\left(\mathbb{R}_{+}, Y\right)$, then $P^{i} y$ is the function in $L_{\text {loc }}^{2}\left(\mathbb{R}_{+}, Y^{i}\right)$ given by $\left(P^{i} y\right)(t)=$ $P^{i} y(t)$. Similarly, for $u \in L_{\mathrm{loc}}^{2}\left(\mathbb{R}_{+}, U^{i}\right)$, the symbol $E^{i} u$ denotes the function in $L_{\text {loc }}^{2}\left(\mathbb{R}_{+}, U\right)$ given by $\left(E^{i} u\right)(t)=E^{i} u(t)$. The decompositions of the input and output spaces, $U=U^{1} \times U^{2}$ and $Y=Y^{1} \times Y^{2}$, respectively, induce four well-posed systems, namely

$$
\Sigma^{i j}:=\left(\mathbb{T}, \Phi E^{j}, P^{i} \Psi, P^{i} \mathbb{G} E^{j}\right), \quad i, j=1,2 .
$$


Obviously, the state, input and output spaces of $\Sigma^{i j}$ are given by $X, U^{j}$ and $Y^{i}$, respectively. For $K^{i j} \in \mathcal{L}\left(Y^{j}, U^{i}\right)$, let $K \in \mathcal{L}(Y, U)$ be defined by

$$
K y=E^{i} K^{i j} P^{j} y \quad \forall y \in Y .
$$

For example, if $i=1$ and $j=2$, then

$$
K=\left(\begin{array}{cc}
0 & K^{12} \\
0 & 0
\end{array}\right)
$$

\section{Incremental stability of infinite-dimensional Lur'e systems}

We start this section by defining the class of Lur'e systems which we will be considering, thereby formalizing the arrangement depicted in Fig. 1. Given an initial state $x^{0}$ and an input $u \in L_{\text {loc }}^{2}\left(\mathbb{R}_{+}, U\right)$, the corresponding state and output trajectories of $\Sigma$ are given by (2.2). Let $i, j \in\{1,2\}$ and let $f: Y^{j} \rightarrow U^{i}$ be a nonlinearity. The closed-loop system obtained by applying the feedback

$$
u=E^{i}\left(f \circ P^{j} y\right)+v, \quad \text { where } v \in L_{\mathrm{loc}}^{2}\left(\mathbb{R}_{+}, U\right),
$$

is then given by

$$
\left.\begin{array}{l}
x(t)=\mathbb{T}_{t} x^{0}+\Phi_{t} \mathbf{P}_{t}\left(E^{i}\left(f \circ P^{j} y\right)+v\right), \\
\mathbf{P}_{t} y=\Psi_{t} x^{0}+\mathbb{G}_{t} \mathbf{P}_{t}\left(E^{i}\left(f \circ P^{j} y\right)+v\right) .
\end{array}\right\}
$$

As an illustration, Fig. 1 corresponds to the case $i=j=2$. Given $x^{0} \in X$ and $v \in L_{\text {loc }}^{2}\left(\mathbb{R}_{+}, U\right)$, a solution of the Lur'e system (3.1) on $[0, \sigma)$, where $0<\sigma \leq \infty$, is a pair $(x, y) \in C([0, \sigma), X) \times L_{\text {loc }}^{2}([0, \sigma), Y)$ such that $f \circ P^{j} y \in L_{\text {loc }}^{2}\left([0, \sigma), U^{i}\right)$ and (3.1) holds for all $t \in[0, \sigma)$. Obviously, if $(x, y)$ is a solution of (3.1), then $x(0)=x^{0}$.

It can be shown (by invoking Zorn's lemma) that, for every solution of (3.1) on $[0, \sigma)$, there exists a maximally defined solution (3.1) defined on $[0, \tau)$ with $\sigma \leq \tau \leq$ $\infty$ which cannot be extended any further (that is, $\tau$ is maximal).

The set of all triples $(v, x, y)$ in $L_{\text {loc }}^{2}\left(\mathbb{R}_{+}, U\right) \times C\left(\mathbb{R}_{+}, X\right) \times L_{\text {loc }}^{2}\left(\mathbb{R}_{+}, Y\right)$ such that (3.1) holds with $x^{0}=x(0)$ is said to be the behaviour of (3.1) and is denoted by $\mathcal{B}$. Elements of $\mathcal{B}$ will sometimes be referred to as trajectories of (3.1). In particular, if $(v, x, y) \in \mathcal{B}$, then $(x, y)$ is a solution of (3.1) which is defined on $\mathbb{R}_{+}$and with $x^{0}=$ $x(0)$. In an ISS context, we consider external inputs $v$ which belong to $L_{\text {loc }}^{\infty}\left(\mathbb{R}_{+}, U\right) \subset$ $L_{\text {loc }}^{2}\left(\mathbb{R}_{+}, U\right)$. More generally, for $2 \leq q \leq \infty$, we may wish to consider inputs $v$ in $L_{\text {loc }}^{q}\left(\mathbb{R}_{+}, U\right) \subset L_{\text {loc }}^{2}\left(\mathbb{R}_{+}, U\right)$. It is therefore convenient to define the following "sub-behaviour" of $\mathcal{B}$ :

$$
\mathcal{B}^{q}:=\left\{(v, x, y) \in \mathcal{B}: v \in L_{\text {loc }}^{q}\left(\mathbb{R}_{+}, U\right)\right\} .
$$


Obviously, we have $\mathcal{B}^{2}=\mathcal{B}$. A key property of the behaviour $\mathcal{B}^{q}$ is its invariance with respect to left translations, that is,

$$
(v, x, y) \in \mathcal{B}^{q} \quad \Longrightarrow \quad\left(\mathbf{S}_{\tau} v, \mathbf{S}_{\tau} x, \mathbf{S}_{\tau} y\right) \in \mathcal{B}^{q} \quad \forall \tau \geq 0
$$

In this paper, we are mainly concerned with stability and convergence properties of (3.1) and not with existence and uniqueness questions. However, we state a simple, but important, existence and uniqueness result from [43].

Proposition 3.1 If $f: Y^{j} \rightarrow U^{i}$ is globally Lipschitz with Lipschitz constant $\lambda \geq 0$ and

$$
\lambda \liminf _{\alpha \rightarrow \infty}\left\|P^{j} \mathbf{G} E^{i}\right\|_{H_{\alpha}^{\infty}}<1
$$

then, for all $x^{0} \in X$ and $v \in L_{\text {loc }}^{2}\left(\mathbb{R}_{+}, U\right)$, the Lur'e system (3.1) has a unique solution on $\mathbb{R}_{+}$.

For later purposes, we define the bi-lateral behaviour $\mathcal{B B}$ of (3.1) as the set of all triples $(v, x, y) \in L_{\text {loc }}^{2}(\mathbb{R}, U) \times C(\mathbb{R}, X) \times L_{\text {loc }}^{2}(\mathbb{R}, Y)$ such that, for every $t_{0} \in \mathbb{R}$,

$$
\left.\begin{array}{rl}
x(t) & =\mathbb{T}_{t-t_{0}} x\left(t_{0}\right)+\Phi_{t-t_{0}} \mathbf{P}_{t-t_{0}}\left(E^{i}\left(f \circ P^{j} \mathbf{S}_{t_{0}} y\right)+\mathbf{S}_{t_{0}} v\right) \\
\mathbf{P}_{t-t_{0}} \mathbf{S}_{t_{0}} y & =\Psi_{t-t_{0}} x\left(t_{0}\right)+\mathbb{G}_{t-t_{0}} \mathbf{P}_{t-t_{0}}\left(E^{i}\left(f \circ P^{j} \mathbf{S}_{t_{0}} y\right)+\mathbf{S}_{t_{0}} v\right)
\end{array}\right\} \forall t \geq t_{0} .
$$

We refer to the elements of $\mathcal{B B}$ as bi-trajectories of (3.1). Obviously, a bi-trajectory restricted to $\mathbb{R}_{+}$is an element in $\mathcal{B}$. Furthermore, the bi-lateral behaviour $\mathcal{B B}$ is invariant with respect to all translations, that is,

$$
(v, x, y) \in \mathcal{B B} \Longrightarrow\left(\mathbf{S}_{\tau} v, \mathbf{S}_{\tau} x, \mathbf{S}_{\tau} y\right) \in \mathcal{B B} \quad \forall \tau \in \mathbb{R}
$$

The next lemma (which can be found in [16]) shows that the behaviour $\mathcal{B}$ of (3.1) is identical to the behaviour of the feedback interconnection obtained when the linear system $\Sigma^{K}$ is subjected to the feedback law $u=E^{i} f\left(P^{j} y\right)-K y+v$, where $K \in$ $\mathcal{L}(Y, U)$ is an admissible feedback operator for $\Sigma$.

Lemma 3.2 Let $K \in \mathcal{L}(Y, U)$ be an admissible feedback operator for $\Sigma$ and let $(v, x, y) \in L_{\text {loc }}^{2}\left(\mathbb{R}_{+}, U\right) \times C\left(\mathbb{R}_{+}, X\right) \times L_{\text {loc }}^{2}\left(\mathbb{R}_{+}, Y\right)$. The triple $(v, x, y)$ is in $\mathcal{B}$ if, and only if,

$$
\left.\begin{array}{rl}
x(t) & =\mathbb{T}_{t}^{K} x(0)+\Phi_{t}^{K} \mathbf{P}_{t}\left(E^{i}\left(f \circ P^{j} y\right)+v-K y\right) \\
\mathbf{P}_{t} y & =\Psi_{t}^{K} x(0)+\mathbb{G}_{t}^{K} \mathbf{P}_{t}\left(E^{i}\left(f \circ P^{j} y\right)+v-K y\right)
\end{array}\right\} \quad \forall t \geq 0 .
$$

A triple $\left(v^{\mathrm{eq}}, x^{\mathrm{eq}}, y^{\mathrm{eq}}\right) \in U \times X \times Y$ is said to be an equilibrium or equilibrium triple of the Lur'e system (3.1) if the constant trajectory $t \mapsto\left(v^{\mathrm{eq}}, x^{\mathrm{eq}}, y^{\mathrm{eq}}\right)$ belongs to $\mathcal{B}$ (in which case it is also a bi-trajectory). The next result provides formulas relating the components of an equilibrium triple $\left(v^{\mathrm{eq}}, x^{\mathrm{eq}}, y^{\mathrm{eq}}\right)$. 
Proposition 3.3 Let $\left(v^{\mathrm{eq}}, x^{\mathrm{eq}}, y^{\mathrm{eq}}\right) \in U \times X \times Y$, let $\eta \in \mathbb{C}$ such that $\operatorname{Re} \eta>\omega(\mathbb{T})$ and set $u^{\mathrm{eq}}:=E^{i} f\left(P^{j} y^{\mathrm{eq}}\right)+v^{\mathrm{eq}}$. The triple $\left(v^{\mathrm{eq}}, x^{\mathrm{eq}}, y^{\mathrm{eq}}\right)$ is an equilibrium of (3.1) if, and only if,

$$
A x^{\mathrm{eq}}+B u^{\mathrm{eq}}=0 \text { and } y^{\mathrm{eq}}=C\left(x^{\mathrm{eq}}-(\eta I-A)^{-1} B u^{\mathrm{eq}}\right)+\mathbf{G}(\eta) u^{\mathrm{eq}} .
$$

In particular, $\left(-E^{i} f(0), 0,0\right)$ is an equilibrium triple of $(3.1)$.

We refer to [16] for a proof of Proposition 3.3.

Note that the identity $A x^{\mathrm{eq}}+B u^{\mathrm{eq}}=0$ implies that $x^{\mathrm{eq}}-(\eta I-A)^{-1} B u^{\mathrm{eq}} \in X_{1}$ and thus, the expression $C\left(x^{\mathrm{eq}}-(\eta I-A)^{-1} B u^{\mathrm{eq}}\right)$ is well defined.

Let $2 \leq q \leq \infty$. An equilibrium triple ( $\left.v^{\mathrm{eq}}, x^{\mathrm{eq}}, y^{\mathrm{eq}}\right)$ of (3.1) is said to be exponentially $L^{q}$-input-to-state stable (exponentially $L^{q}-I S S$ ) if there exist positive constants $\Gamma$ and $\gamma$ such that

$$
\left\|x(t)-x^{\mathrm{eq}}\right\| \leq \Gamma\left(e^{-\gamma t}\left\|x(0)-x^{\mathrm{eq}}\right\|+\left\|\mathbf{P}_{t}\left(v-v^{\mathrm{eq}} \vartheta\right)\right\|_{L^{q}}\right) \quad \forall t \geq 0, \quad \forall(v, x, y) \in \mathcal{B}^{q},
$$

where $\vartheta(t)=1$ for all $t \geq 0$. Furthermore, (3.1) is said to be exponentially incrementally $L^{q}$-input-to-state stable (exponentially $L^{q}-\delta I S S$ ) if there exist positive constants $\Gamma$ and $\gamma>0$ such that, for all $\left(v_{1}, x_{1}, y_{1}\right),\left(v_{2}, x_{2}, y_{2}\right) \in \mathcal{B}^{q}$,

$$
\left\|x_{1}(t)-x_{2}(t)\right\| \leq \Gamma\left(e^{-\gamma t}\left\|x_{1}(0)-x_{2}(0)\right\|+\left\|\mathbf{P}_{t}\left(v_{1}-v_{2}\right)\right\|_{L^{q}}\right) \quad \forall t \geq 0 .
$$

Here $v_{i}$ and $y_{i}$ should not be confused with $v^{i}$ and $y^{i}, i=1,2$, which appear in (1.1) and Fig. 1.

We introduce a further type of "sub-behaviour" which shall be useful in formulating our stability results. For a non-empty subset $Z \subset Y^{j}$ and $2 \leq q \leq \infty$, we set

$$
\mathcal{B}_{Z}^{q}:=\left\{(v, x, y) \in \mathcal{B}^{q}: P^{j} y(t) \in Z \text { for a.e } t \geq 0\right\}
$$

Furthermore, $\mathcal{B}_{Z}:=\mathcal{B}_{Z}^{2}$.

The following theorem, a refinement of [16, Theorem 4.1], is reminiscent of the complex Aizerman conjecture in finite dimensions (which is known to be true, see $[19,20,22])$ : incremental stability properties of the nonlinear system (3.1) are guaranteed by the assumption that a corresponding linear feedback system is stable for all linear complex feedback operators belonging to a certain ball, provided the nonlinearity satisfies, in a suitable and natural sense, an incremental version of the same boundedness condition. Before we state the results, it is convenient to define

$$
\Delta:=\left\{(t, s) \in \mathbb{R}^{2}: t \geq s \geq 0\right\} \subset \mathbb{R}_{+}^{2} .
$$

Theorem 3.4 Let $\Sigma=(\mathbb{T}, \Phi, \Psi, \mathbb{G})$ be a well-posed linear system, let $i, j \in\{1,2\}$, $K^{i j} \in \mathcal{L}\left(Y^{j}, U^{i}\right), r>0$, and let $Z_{1}, Z_{2} \subset Y^{j}$ be non-empty subsets. Assume that 
$\Sigma^{j i}=\left(\mathbb{T}, \Phi E^{i}, P^{j} \Psi, P^{j} \mathbb{G} E^{i}\right)$ is optimizable and estimatable and $\mathbb{B}\left(K^{i j}, r\right) \subset$ $\mathbb{S}\left(P^{j} \mathbf{G} E^{i}\right)$. If $f: Y^{j} \rightarrow U^{i}$ satisfies

$$
\sup _{\left(z_{1}, z_{2}\right) \in Z_{1} \times Z_{2}, z_{1} \neq z_{2}} \frac{\left\|f\left(z_{1}\right)-f\left(z_{2}\right)-K^{i j}\left(z_{1}-z_{2}\right)\right\|}{\left\|z_{1}-z_{2}\right\|}<r,
$$

then the following statements hold.

(1) For every $2 \leq q \leq \infty$, there exist constants $\Gamma_{q}>0$ and $\gamma>0$ such that, for all $\left(v_{1}, x_{1}, y_{1}\right) \in \mathcal{B}_{Z_{1}}^{q}$ and all $\left(v_{2}, x_{2}, y_{2}\right) \in \mathcal{B}_{Z_{2}}^{q}$,

$$
\begin{aligned}
\left\|x_{1}(t)-x_{2}(t)\right\| \leq & \Gamma_{q}\left(e^{-\gamma\left(t-t_{0}\right)}\left\|x_{1}\left(t_{0}\right)-x_{2}\left(t_{0}\right)\right\|\right. \\
& \left.+\left\|v_{1}-v_{2}\right\|_{L^{q}\left(t_{0}, t\right)}\right) \quad \forall\left(t, t_{0}\right) \in \Delta .
\end{aligned}
$$

Here, $\Gamma_{q}$ depends on $q$, but $\gamma$ does not. If $v_{1}-v_{2} \in L_{\alpha}^{q}\left(\mathbb{R}_{+}, U\right)$ for some $\alpha>0$, then $\left(x_{1}(t)-x_{2}(t)\right) \rightarrow 0$ exponentially fast as $t \rightarrow \infty$. Furthermore, if $v_{1}-v_{2} \in L^{q}\left(\mathbb{R}_{+}, U\right)$ for some $1 \leq q<\infty$ or if $v_{1}-v_{2} \in L^{\infty}\left(\mathbb{R}_{+}, U\right)$ and $\lim _{t \rightarrow \infty}\left\|\mathbf{S}_{t}\left(v_{1}-v_{2}\right)\right\|_{L^{\infty}}=0$, then $\left(x_{1}(t)-x_{2}(t)\right) \rightarrow 0$ as $t \rightarrow \infty$.

(2) There exist constants $\Gamma>0$ and $\varepsilon>0$ such that, for all $\left(v_{1}, x_{1}, y_{1}\right) \in \mathcal{B}_{Z_{1}}$, all $\left(v_{2}, x_{2}, y_{2}\right) \in \mathcal{B}_{Z_{2}}$ and all $\alpha \in[0, \varepsilon]$,

$$
\begin{aligned}
& \left\|x_{1}-x_{2}\right\|_{L_{\alpha}^{2}\left(t_{0}, t\right)}+\left\|y_{1}-y_{2}\right\|_{L_{\alpha}^{2}\left(t_{0}, t\right)} \\
& \quad \leq \Gamma\left(\left\|x_{1}\left(t_{0}\right)-x_{2}\left(t_{0}\right)\right\|+\left\|v_{1}-v_{2}\right\|_{L_{\alpha}^{2}\left(t_{0}, t\right)}\right) \quad \forall\left(t, t_{0}\right) \in \Delta .
\end{aligned}
$$

(3) There exist constants $\tilde{\Gamma}_{2}>0$ and $\tilde{\gamma}>0$ such that, for all $\left(v_{1}, x_{1}, y_{1}\right) \in \mathcal{B}_{Z_{1}}$ and all $\left(v_{2}, x_{2}, y_{2}\right) \in \mathcal{B}_{Z_{2}}$ with $v_{1}-v_{2} \in U L_{\text {loc }}^{2}\left(\mathbb{R}_{+}, U\right)$

$$
\begin{aligned}
\left\|x_{1}(t)-x_{2}(t)\right\| \leq & \tilde{\Gamma}_{2}\left(e^{-\tilde{\gamma}\left(t-t_{0}\right)}\left\|x_{1}\left(t_{0}\right)-x_{2}\left(t_{0}\right)\right\|\right. \\
& \left.+\left\|\mathbf{S}_{t_{0}}\left(v_{1}-v_{2}\right)\right\|_{S^{2}}\right) \quad \forall\left(t, t_{0}\right) \in \Delta .
\end{aligned}
$$

If $v_{1}-v_{2} \in U_{\alpha} L_{\text {loc }}^{2}\left(\mathbb{R}_{+}, U\right)$ for some $\alpha \geq 0$, then $\left(x_{1}(t)-x_{2}(t)\right) \rightarrow 0$ as $t \rightarrow \infty$ and the convergence is exponentially fast if $\alpha>0$.

(4) There exists a constant $\tilde{\Gamma}>0$ such that, for all $\left(v_{1}, x_{1}, y_{1}\right) \in \mathcal{B}_{Z_{1}}$ and all $\left(v_{2}, x_{2}, y_{2}\right) \in \mathcal{B}_{Z_{2}}$ with $v_{1}-v_{2} \in U L_{\text {loc }}^{2}\left(\mathbb{R}_{+}, U\right)$,

$$
\begin{aligned}
& \left\|\mathbf{S}_{t_{0}}\left(x_{1}-x_{2}\right)\right\|_{S^{2}}+\left\|\mathbf{S}_{t_{0}}\left(y_{1}-y_{2}\right)\right\|_{S^{2}} \\
& \quad \leq \tilde{\Gamma}\left(\left\|x_{1}\left(t_{0}\right)-x_{2}\left(t_{0}\right)\right\|+\left\|\mathbf{S}_{t_{0}}\left(v_{1}-v_{2}\right)\right\|_{S^{2}}\right) \quad \forall t_{0} \geq 0 .
\end{aligned}
$$

If $v_{1}-v_{2} \in U_{\alpha} L_{\text {loc }}^{2}\left(\mathbb{R}_{+}, U\right)$ for some $\alpha \geq 0$, then $\mathbf{S}_{t}\left(x_{1}-x_{2}\right) \rightarrow 0$ and $\mathbf{S}_{t}\left(y_{1}-y_{2}\right) \rightarrow 0$ as $t \rightarrow \infty$ and, in each case, the convergence is exponentially fast if $\alpha>0$.

It follows from (2.1) that if in statement (3), $v_{1}-v_{2} \in U L_{\text {loc }}^{q}\left(\mathbb{R}_{+}, U\right)$ for $q \in(2, \infty)$, then (3.4) holds with $\left\|\mathbf{S}_{t_{0}}\left(v_{1}-v_{2}\right)\right\|_{S^{2}}$ replaced by $\left\|\mathbf{S}_{t_{0}}\left(v_{1}-v_{2}\right)\right\|_{S^{q}}$. 
As for the role of the sets $Z_{1}$ and $Z_{2}$ in Theorem 3.4, we highlight two important special cases.

Special case 1 Assume that ( $\left.v^{\mathrm{eq}}, x^{\mathrm{eq}}, y^{\mathrm{eq}}\right) \in U \times X \times Y$ is an equilibrium triple of the Lur'e system (3.1) and the assumptions of Theorem 3.4 hold with $Z_{1}=Y^{j}$ and $Z_{2}=\left\{P^{j} y^{\mathrm{eq}}\right\}$. Then, the constant trajectory $\left(v^{\mathrm{eq}}, x^{\mathrm{eq}}, y^{\mathrm{eq}}\right)$ is in $\mathcal{B}_{Z_{2}}^{\infty}$ and statement (1) implies that, for every $2 \leq q \leq \infty$, the equilibrium $\left(v^{\mathrm{eq}}, x^{\mathrm{eq}}, y^{\mathrm{eq}}\right)$ is exponentially $L^{q}$-ISS. Furthermore, statement (3) guarantees that, for any $(v, x, y) \in \mathcal{B}^{q}$ with $v \in$ $U L_{\mathrm{loc}}^{q}\left(\mathbb{R}_{+}, U\right)$ and $2 \leq q<\infty$, the state $x$ is bounded.

Special case 2 Assume that the hypotheses of Theorem 3.4 hold with $Z_{1}=Z_{2}=Y^{j}$ (and so (3.2) is equivalent to $z \mapsto f(z)-K^{i j} z$ being globally Lipschitz with Lipschitz constant smaller than $r$ ). In this case, statement (1) of Theorem 3.4 implies that the Lur'e system (3.1) is exponentially $L^{q}$ - $\delta$ ISS for every $q$ such that $2 \leq q \leq \infty$. Furthermore, as a consequence of Proposition 3.1 and Lemma 3.2, for every pair $\left(x^{0}, v\right) \in X \times L_{\text {loc }}^{2}\left(\mathbb{R}_{+}, U\right)$, there exists a unique triple $(v, x, y) \in \mathcal{B}$ such that $x(0)=x^{0}$.

As compared to [16, Theorem 4.1], the new contribution of Theorem 3.4 are statements (3) and (4) which provide bounds in terms of the Stepanov norm of $\mathbf{S}_{t_{0}}\left(v_{1}-v_{2}\right)$.

Proof of Theorem 3.4 To prove statement (1), let $\left(v_{1}, x_{1}, y_{1}\right) \in \mathcal{B}_{Z_{1}}^{q}$ and $\left(v_{2}, x_{2}, y_{2}\right) \in$ $\mathcal{B}_{Z_{2}}^{q}$ and note that, for any $t_{0} \geq 0,\left(\mathbf{S}_{t_{0}} v_{1}, \mathbf{S}_{t_{0}} x_{1}, \mathbf{S}_{t_{0}} y_{1}\right) \in \mathcal{B}_{Z_{1}}^{q}$ and $\left(\mathbf{S}_{t_{0}} v_{2}, \mathbf{S}_{t_{0}} x_{2}, \mathbf{S}_{t_{0}} y_{2}\right) \in$ $\mathcal{B}_{Z_{2}}^{q}$, and thus, by [16, Theorem 4.1], there exist constants $\Gamma_{q}>0$ and $\gamma>0$, such that

$$
\begin{aligned}
& \left\|\left(\mathbf{S}_{t_{0}} x_{1}\right)(s)-\left(\mathbf{S}_{t_{0}} x_{2}\right)(s)\right\| \\
& \quad \leq \Gamma_{q}\left(e^{-\gamma s}\left\|\left(\mathbf{S}_{t_{0}} x_{1}\right)(0)-\left(\mathbf{S}_{t_{0}} x_{2}\right)(0)\right\|+\left\|\mathbf{P}_{s}\left(\mathbf{S}_{t_{0}} v_{1}-\mathbf{S}_{t_{0}} v_{2}\right)\right\|_{L^{q}}\right) \quad \forall s \geq 0 .
\end{aligned}
$$

Setting $t:=s+t_{0} \geq 0$, it follows that

$$
\left\|x_{1}(t)-x_{2}(t)\right\| \leq \Gamma_{q}\left(e^{-\gamma\left(t-t_{0}\right)}\left\|x_{1}\left(t_{0}\right)-x_{2}\left(t_{0}\right)\right\|+\left\|v_{1}-v_{2}\right\|_{L^{q}\left(t_{0}, t\right)}\right) \quad \forall t \geq t_{0},
$$

which is (3.3). Assume now that $v_{1}-v_{2} \in L_{\alpha}^{q}\left(\mathbb{R}_{+}, U\right)$ for some $\alpha>0$. Then, by (3.3), $x_{1}-x_{2}$ is bounded and, as $\left\|v_{1}-v_{2}\right\|_{L^{q}\left(t_{0}, t\right)} \leq e^{-\alpha t_{0}} \mid v_{1}-v_{2} \|_{L_{\alpha}^{q}\left(t_{0}, t\right)}$ for all $\left(t, t_{0}\right) \in \Delta$, another application of (3.3) (with $t_{0}=t / 2$ ) yields

$$
\left\|x_{1}(t)-x_{2}(t)\right\| \leq \Gamma_{q}\left(e^{-\gamma t / 2}\left\|x_{1}-x_{2}\right\|_{\infty}+e^{-\alpha t / 2}\left\|v_{1}-v_{2}\right\|_{L_{\alpha}^{q}(t / 2, \infty)}\right) \quad \forall t \geq 0,
$$

showing that $x_{1}(t)-x_{2}(t)$ converges exponentially fast to 0 as $t \rightarrow \infty$. The remaining convergence claim in statement (1) can be proved by a similar argument.

As in the derivation of (3.3), we can use [16, Theorem 4.1] and the left-translation invariance of $\mathcal{B}_{Z_{1}}$ and $\mathcal{B}_{Z_{2}}$ to prove statement (2). The details are left to the reader.

We proceed to prove statement (3). Let $t_{0} \geq 0,\left(v_{1}, x_{1}, y_{1}\right) \in \mathcal{B}_{Z_{1}}$ and $\left(v_{2}, x_{2}, y_{2}\right) \in$ $\mathcal{B}_{Z_{2}}$ with $v_{1}-v_{2} \in U L_{\text {loc }}^{2}\left(\mathbb{R}_{+}, U\right)$. Setting $x:=x_{1}-x_{2}$ and $v:=v_{1}-v_{2}$, we obtain from statement (1) that 


$$
\|x(t)\| \leq \Gamma_{2}\left(e^{-\gamma(t-s)}\|x(s)\|+\|v\|_{L^{q}(s, t)}\right) \quad \forall(t, s) \in \Delta .
$$

Choose $\tau>0$ such that $\Gamma_{2} e^{-\gamma \tau}<1$ and let $m$ be the smallest integer such that $m \geq \tau$. A straightforward argument shows that

$$
\|v\|_{L^{2}(a, a+\tau)} \leq \sqrt{m}\left\|\mathbf{S}_{t_{0}} v\right\|_{S^{2}} \quad \forall\left(a, t_{0}\right) \in \Delta
$$

In particular,

$$
b:=\sup _{k \in \mathbb{Z}_{+}}\|v\|_{L^{q}\left(t_{0}+k \tau, t_{0}+(k+1) \tau\right)} \leq \sqrt{m}\left\|\mathbf{S}_{t_{0}} v\right\|_{S^{2}}
$$

and so, by (3.6) with $t=t_{0}+(k+1) \tau$ and $s=t_{0}+k \tau$,

$\left\|x\left(t_{0}+(k+1) \tau\right)\right\| \leq \Gamma_{2}\left(e^{-\gamma \tau}\left\|x\left(t_{0}+k \tau\right)\right\|+b\right) \leq \theta\left\|x\left(t_{0}+k \tau\right)\right\|+\Gamma_{2} b \quad \forall k \in \mathbb{Z}_{+}$, where $\theta:=\Gamma_{2} e^{-\gamma \tau}<1$. Consequently,

$$
\left\|x\left(t_{0}+k \tau\right)\right\| \leq \theta^{k}\left\|x\left(t_{0}\right)\right\|+\Gamma_{2} b \sum_{j=0}^{k-1} \theta^{j} \leq \theta^{k}\left\|x\left(t_{0}\right)\right\|+\frac{\Gamma_{2} b}{1-\theta} \quad \forall k \in \mathbb{N}
$$

Appealing to (3.6) with $s=t_{0}+k \tau$ we obtain

$\|x(t)\| \leq \Gamma_{2}\left(\left\|x\left(t_{0}+k \tau\right)\right\|+\|v\|_{L^{2}\left(t_{0}+k \tau, t\right)}\right) \quad \forall t \in\left[t_{0}+k \tau, t_{0}+(k+1) \tau\right], \quad \forall k \in \mathbb{Z}_{+}$.

Now $\|v\|_{L^{2}\left(t_{0}+k \tau, t\right)} \leq b$ for all $t \in\left[t_{0}+k \tau, t_{0}+(k+1) \tau\right]$ and all $k \in \mathbb{Z}_{+}$, and so, invoking (3.7),

$\|x(t)\| \leq \Gamma_{2}\left(\theta^{k}\left\|x\left(t_{0}\right)\right\|+\left(\Gamma_{2}+1-\theta\right) b /(1-\theta)\right) \quad \forall t \in\left[t_{0}+k \tau, t_{0}+(k+1) \tau\right], \quad \forall k \in \mathbb{Z}_{+}$.

Consequently, setting $\tilde{\gamma}:=-(\ln \theta) / \tau>0$, we have that

$$
\|x(t)\| \leq \tilde{\Gamma}_{2}\left(e^{-\tilde{\gamma}\left(t-t_{0}\right)}\left\|x\left(t_{0}\right)\right\|+\left\|\mathbf{S}_{t_{0}} v\right\|_{S^{2}}\right) \quad \forall\left(t, t_{0}\right) \in \Delta
$$

where $\tilde{\Gamma}_{2}:=\Gamma_{2} \max \left(e^{\tilde{\gamma} \tau}, \sqrt{m}\left(\Gamma_{2}+1-\theta\right) /(1-\theta)\right)$, establishing (3.4). Now assume that $v_{1}-v_{2} \in U_{\alpha} L_{\text {loc }}^{2}\left(\mathbb{R}_{+}, U\right)$ for some $\alpha \geq 0$. Then, by (3.4), $x_{1}-x_{2}$ is bounded and, as $e^{\alpha t}\left\|\mathbf{S}_{t}\left(v_{1}-v_{2}\right)\right\|_{S^{2}} \leq\left\|\mathbf{S}_{t}\left(\exp _{\alpha}\left(v_{1}-v_{2}\right)\right)\right\|_{S^{2}}$ for all $t \geq 0$, another application of (3.4) (with $t_{0}=t / 2$ ) yields

$$
\left\|x_{1}(t)-x_{2}(t)\right\| \leq \tilde{\Gamma}_{2}\left(e^{-\tilde{\gamma} t / 2}\left\|x_{1}-x_{2}\right\|_{\infty}+e^{-\alpha t / 2}\left\|\mathbf{S}_{t / 2}\left(\exp _{\alpha}\left(v_{1}-v_{2}\right)\right)\right\|_{S^{2}}\right) \quad \forall t \geq 0
$$

showing that $x_{1}(t)-x_{2}(t)$ converges to 0 as $t \rightarrow \infty$ and that the convergence is exponentially fast if $\alpha>0$, completing the proof of statement (3). 
To prove statement (4), let $\left(v_{1}, x_{1}, y_{1}\right) \in \mathcal{B}_{Z_{1}}$ and $\left(v_{2}, x_{2}, y_{2}\right) \in \mathcal{B}_{Z_{2}}$ with $v_{1}-v_{2} \in$ $U L_{\text {loc }}^{2}\left(\mathbb{R}_{+}, U\right)$. Then, for every $t_{0} \geq 0$,

$$
\left(\mathbf{S}_{t_{0}} v_{j}, \mathbf{S}_{t_{0}} x_{j}, \mathbf{S}_{t_{0}} y_{j}\right) \in \mathcal{B}_{Z_{j}}, \quad j=1,2
$$

Therefore, setting $v:=v_{1}-v_{2}, x:=x_{1}-x_{2}$ and $y:=y_{1}-y_{2}$, we obtain from statement (2) that

$$
\left\|\mathbf{S}_{t_{0}} x\right\|_{L^{2}(\tau, \tau+1)}+\left\|\mathbf{S}_{t_{0}} y\right\|_{L^{2}(\tau, \tau+1)} \leq \Gamma\left(\left\|\left(\mathbf{S}_{t_{0}} x\right)(\tau)\right\|+\left\|\mathbf{S}_{t_{0}} v\right\|_{L^{2}(\tau, \tau+1)}\right) \quad \forall \tau, t_{0} \geq 0
$$

The above inequality implies that, for every $t_{0} \geq 0$,

$$
\left\|\mathbf{S}_{t_{0}} x\right\|_{S^{2}}+\left\|\mathbf{S}_{t_{0}} y\right\|_{S^{2}} \leq 2 \Gamma\left(\sup _{\tau \geq 0}\left\|\left(\mathbf{S}_{t_{0}} x\right)(\tau)\right\|+\left\|\mathbf{S}_{t_{0}} v\right\|_{S^{2}}\right) .
$$

Now, as $\left(\mathbf{S}_{t_{0}} v_{j}, \mathbf{S}_{t_{0}} x_{j}, \mathbf{S}_{t_{0}} y_{j}\right) \in \mathcal{B}_{Z_{j}}, j=1,2$, an application of statement (3) yields,

$$
\left\|\left(\mathbf{S}_{t_{0}} x\right)(s)\right\| \leq \tilde{\Gamma}_{2}\left(e^{-\tilde{\gamma} s}\left\|\left(\mathbf{S}_{t_{0}} x\right)(0)\right\|+\left\|\mathbf{S}_{t_{0}} v\right\|_{S^{2}}\right) \quad \forall s, t_{0} \geq 0
$$

and thus, for every $t_{0} \geq 0$,

$$
\left\|\mathbf{S}_{t_{0}} x\right\|_{S^{2}}+\left\|\mathbf{S}_{t_{0}} y\right\|_{S^{2}} \leq \tilde{\Gamma}\left(\left\|x\left(t_{0}\right)\right\|+\left\|\mathbf{S}_{t_{0}} v\right\|_{S^{2}}\right)
$$

where $\tilde{\Gamma}:=2 \Gamma\left(\tilde{\Gamma}_{2}+1\right)$, establishing (3.5). Finally, assume that $v_{1}-v_{2} \in$ $U_{\alpha} L_{\text {loc }}^{2}\left(\mathbb{R}_{+}, U\right)$ for some $\alpha \geq 0$. Then, $e^{\alpha t}\left\|\mathbf{S}_{t}\left(v_{1}-v_{2}\right)\right\|_{S^{2}} \leq\left\|\mathbf{S}_{t}\left(\exp _{\alpha}\left(v_{1}-v_{2}\right)\right)\right\|_{S^{2}}$ for all $t \geq 0$, and invoking statement (3) and (3.5) (with $t_{0}=t / 2$ ) shows that $\mathbf{S}_{t}\left(x_{1}-x_{1}\right)$ and $\mathbf{S}_{t}\left(y_{1}-y_{2}\right)$ converge to 0 as $t \rightarrow \infty$ and that, in each case, the convergence is exponentially fast if $\alpha>0$, completing the proof.

Assuming that $K^{i j} \in \mathbb{S}\left(P^{j} \mathbf{G} E^{i}\right)$ and setting $r:=1 /\left\|\left(P^{j} \mathbf{G} E^{i}\right)^{K^{i j}}\right\|_{H^{\infty}}$, it follows from Lemma 2.1 that $\mathbb{B}\left(K^{i j}, r\right) \subset \mathbb{S}\left(P^{j} \mathbf{G} E^{i}\right)$, and hence, the following small-gain result is an immediate consequence of Theorem 3.4.

Corollary 3.5 Let $\Sigma, Z_{1}$ and $Z_{2}$ be as in Theorem 3.4, let $i, j \in\{1,2\}$ and let $K^{i j} \in \mathbb{S}\left(P^{j} \mathbf{G} E^{i}\right)$. Assume that $\Sigma^{j i}=\left(\mathbb{T}, \Phi E^{i}, P^{j} \Psi, P^{j} \mathbb{G} E^{i}\right)$ is optimizable and estimatable. If $f: Y^{j} \rightarrow U^{i}$ satisfies

$$
\begin{aligned}
& \sup _{\left(z_{1}, z_{2}\right) \in Z_{1} \times Z_{2}, z_{1} \neq z_{2}} \frac{\left\|f\left(z_{1}\right)-f\left(z_{2}\right)-K^{i j}\left(z_{1}-z_{2}\right)\right\|}{\left\|z_{1}-z_{2}\right\|} \\
& \cdot\left\|\left(P^{j} \mathbf{G} E^{i}\right)^{K^{i j}}\right\|_{H^{\infty}<1,}
\end{aligned}
$$

then statements (1)-(4) of Theorem 3.4 hold.

Let $H$ be a complex Hilbert space. We say that $\mathbf{H}: \mathbb{C}_{0} \rightarrow \mathcal{L}(H)$ is positive real if $\mathbf{H}$ is holomorphic with the exception of isolated singularities and $\mathbf{H}(s)+\mathbf{H}^{*}(s)$ is positive 
semidefinite for all $s \in \mathbb{C}_{0}$ which are not singularities of $\mathbf{H}$. In fact, if $\mathbf{H}$ is positive real, then $\mathbf{H}$ is holomorphic on $\mathbb{C}_{0}$ [15, Proposition 3.3].

The following result can be considered as an incremental version of the circle criterion.

Corollary 3.6 Let $\Sigma, Z_{1}$ and $Z_{2}$ be as in Theorem 3.4, let $i, j \in\{1,2\}$ and let $K_{1}, K_{2} \in \mathcal{L}\left(Y^{j}, U^{i}\right)$. Assume that $\Sigma^{j i}=\left(\mathbb{T}, \Phi E^{i}, P^{j} \Psi, P^{j} \mathbb{G} E^{i}\right)$ is optimizable and estimatable, $K_{1}$ is admissible feedback operator for $\Sigma^{j i}$ and $Z_{2}=Y^{j}$. If $\left(I-K_{2} P^{j} \mathbf{G} E^{i}\right)\left(I-K_{1} P^{j} \mathbf{G} E^{i}\right)^{-1}$ is positive real and there exists $\varepsilon>0$ such that $f: Y^{j} \rightarrow U^{i}$ satisfies

$$
\begin{aligned}
& \operatorname{Re}\left\langle f\left(z_{1}\right)-f\left(z_{2}\right)-K_{1}\left(z_{1}-z_{2}\right), f\left(z_{1}\right)-f\left(z_{2}\right)-K_{2}\left(z_{1}-z_{2}\right)\right\rangle \\
& \quad \leq-\varepsilon\left\|z_{1}-z_{2}\right\|^{2} \forall\left(z_{1}, z_{2}\right) \in Z_{1} \times Y^{j}
\end{aligned}
$$

then statements (1)-(4) of Theorem 3.4 hold (with $\left.Z_{2}=Y^{j}\right)$.

The above corollary can be derived from Theorem 3.4 in the same way as [16, Corollary 4.5] is obtained from [16, Theorem 4.1] and we do not repeat the details here.

\section{Lur'e systems with almost periodic inputs}

Before we come to the main result of this paper, we provide some relevant background on almost periodic functions (in the sense of Bohr and its generalization by Stepanov).

Let $R=\mathbb{R}$ or $\mathbb{R}_{+}$and let $W$ be a Banach space. A set $S \subseteq R$ is said to be relatively dense (in $R$ ) if there exists $l>0$ such that

$$
[a, a+l] \cap S \neq \emptyset \quad \forall a \in R .
$$

For $\varepsilon>0$, we say that $\tau \in R$ is an $\varepsilon$-period of $v \in C(R, W)$ if

$$
\|v(t)-v(t+\tau)\| \leq \varepsilon \quad \forall t \in R .
$$

We denote by $P(v, \varepsilon) \subseteq R$ the set of $\varepsilon$-periods of $v$ and we say that $v \in C(R, W)$ is almost periodic (in the sense of Bohr) if $P(v, \varepsilon)$ is relatively dense in $R$ for every $\varepsilon>0$. We denote the set of almost periodic functions $v \in C(R, W)$ by $A P(R, W)$ and mention that $A P(R, W)$ is a closed subspace of $B U C(R, W)$. Obviously, any periodic continuous function is almost periodic.

The straightforward proof of the following lemma is left to the reader.

Lemma 4.1 If $v \in A P(R, W)$, then, for every $\tau \in R$, $\sup _{t \geq \tau}\|v(t)\|=\|v\|_{\infty}$.

The above lemma shows that functions in $A P(R, W)$ are completely determined by their "infinite right tails": if $v, w \in A P(R, W)$ and there exists $\tau \in R$ such that $v(t)=w(t)$ for all $t \geq \tau$, then $v=w$. A similar result holds in the context of "infinite left tails" of almost periodic functions defined on $\mathbb{R}$, but since it is not needed in what follows, we omit the details. 
We say that a function $v \in C\left(\mathbb{R}_{+}, W\right)$ is asymptotically almost periodic if it is of the form $v=v^{\text {ap }}+w$ with $v^{\text {ap }} \in A P\left(\mathbb{R}_{+}, W\right)$ and $w \in C_{0}\left(\mathbb{R}_{+}, W\right)$, where $C_{0}\left(\mathbb{R}_{+}, W\right)$ is the space of functions $u \in C\left(\mathbb{R}_{+}, W\right)$ such that $\lim _{t \rightarrow \infty} u(t)=0$. The space of all asymptotically almost periodic functions is denoted by $A A P\left(\mathbb{R}_{+}, W\right)$, that is,

$$
A A P\left(\mathbb{R}_{+}, W\right)=A P\left(\mathbb{R}_{+}, W\right)+C_{0}\left(\mathbb{R}_{+}, W\right) .
$$

Noting that, by Lemma 4.1,

$$
\|v+w\|_{\infty} \geq\|v\|_{\infty} \quad \forall v \in A P\left(\mathbb{R}_{+}, W\right), \forall w \in C_{0}\left(\mathbb{R}_{+}, W\right),
$$

it is easy to see that $A A P\left(\mathbb{R}_{+}, W\right)$ is a closed subspace of $B U C\left(\mathbb{R}_{+}, W\right)$.

As an immediate consequence of Lemma 4.1, we obtain the following result.

Lemma 4.2 If $v \in A A P\left(\mathbb{R}_{+}, W\right)$, then the decomposition $v=v^{\text {ap }}+w$, where $v^{\text {ap }} \in$ $A P\left(\mathbb{R}_{+}, W\right)$ and $w \in C_{0}\left(\mathbb{R}_{+}, W\right)$, is unique.

In the following, for $v \in A A P\left(\mathbb{R}_{+}, W\right)$, we let $v^{\text {ap }}$ denote the unique function in $A P\left(\mathbb{R}_{+}, W\right)$ such that $v-v^{\text {ap }} \in C_{0}\left(\mathbb{R}_{+}, W\right)$.

It is well known that $v \in C(\mathbb{R}, W)$ is almost periodic if, and only if, the set of translates $\left\{\mathbf{S}_{\tau} v: \tau \in \mathbb{R}\right\}$ is relatively compact in $B C(\mathbb{R}, W)$. Since, for any $v \in C_{0}\left(\mathbb{R}_{+}, W\right)$, the set of left translates $\left\{\mathbf{S}_{\tau} v: \tau \in \mathbb{R}_{+}\right\}$is relatively compact in $B C\left(\mathbb{R}_{+}, W\right)$, it is clear that the above characterization of almost periodicity on $\mathbb{R}$ is not valid for functions in $C\left(\mathbb{R}_{+}, W\right)$. Interestingly, the elements of $A A P\left(\mathbb{R}_{+}, W\right)$ are precisely the functions for which the set $\left\{\mathbf{S}_{\tau} v: \tau \in \mathbb{R}_{+}\right\}$is relatively compact in $B U C\left(\mathbb{R}_{+}, W\right)$, see [28]. For more information on and further characterizations of almost periodicity, we refer the reader to the literature, see, for example, $[1,7,8]$.

There is a close relationship between the spaces $A P\left(\mathbb{R}_{+}, W\right)$ and $A P(\mathbb{R}, W)$ which we now briefly explain. Following an idea in [5, Remark on p. 318], for every $v \in$ $A P\left(\mathbb{R}_{+}, W\right)$, we define a function $v_{\mathrm{e}}: \mathbb{R} \rightarrow W$ by

$$
v_{\mathrm{e}}(t):=\lim _{k \rightarrow \infty} v\left(t+\tau_{k}\right) \quad \forall t \in \mathbb{R}
$$

where $\tau_{k} \in P(v, 1 / k)$ for each $k \in \mathbb{N}$ and $\tau_{k} \rightarrow \infty$ as $k \rightarrow \infty$. For given $t \in \mathbb{R}$, we have

$$
\begin{aligned}
& \left\|v\left(t+\tau_{k}\right)-v\left(t+\tau_{l}\right)\right\| \\
& \quad \leq\left\|v\left(t+\tau_{k}\right)-v\left(t+\tau_{k}+\tau_{l}\right)\right\|+\left\|v\left(t+\tau_{k}+\tau_{l}\right)-v\left(t+\tau_{l}\right)\right\| \leq \frac{1}{l}+\frac{1}{k},
\end{aligned}
$$

for all $k, l \in \mathbb{N}$ sufficiently large, and so $\left(v\left(t+\tau_{k}\right)\right)_{k}$ is a Cauchy sequence. Hence, $v_{\mathrm{e}}(t)$ is well-defined for each $t \in \mathbb{R}$. It is clear that $v_{\mathrm{e}}(t)=v(t)$ for all $t \geq 0$, that is, $v_{\mathrm{e}}$ extends $v$ to $\mathbb{R}$. Furthermore, it is not difficult to show that $v_{\mathrm{e}}$ is continuous and $P\left(v_{\mathrm{e}}, \varepsilon\right)=\{ \pm \tau: \tau \in P(v, \varepsilon)\}$. In particular, $v_{\mathrm{e}} \in A P(\mathbb{R}, W)$. Moreover, there is no other function in $A P(\mathbb{R}, W)$ which extends $v$ to $\mathbb{R}$, and Lemma 4.1 guarantees that

$$
\sup _{t \in \mathbb{R}}\left\|v_{\mathrm{e}}(t)\right\|=\sup _{t \in \mathbb{R}_{+}}\|v(t)\| \text {. }
$$


It is now clear that the map $A P\left(\mathbb{R}_{+}, W\right) \rightarrow A P(\mathbb{R}, W), v \mapsto v_{\mathrm{e}}$ is an isometric isomorphism. We remark that, by invoking the translation semigroup acting on $A P\left(\mathbb{R}_{+}, W\right),[4]$ provides an alternative approach to establishing that every element in $A P\left(\mathbb{R}_{+}, W\right)$ has an almost periodic extension to $\mathbb{R}$.

For a function $v \in A P(\mathbb{R}, W)$, the generalized Fourier coefficients of $v$ are defined by

$$
\hat{v}(\lambda):=\lim _{T \rightarrow \infty} \frac{1}{2 T} \int_{-T}^{T} e^{-i \lambda t} v(t) d t \quad \forall \lambda \in \mathbb{R} .
$$

It is well known that the above limit exists for all $\lambda \in \mathbb{R}$ and the frequency spectrum

$$
\sigma_{\mathrm{f}}(v):=\{\lambda \in \mathbb{R}: \hat{v}(\lambda) \neq 0\}
$$

of $v$ is countable, see, for example, [1,8]. The module $\bmod (v)$ of $v \in A P(\mathbb{R}, W)$ is the set of all numbers of the form $\sum_{\lambda \in \sigma_{\mathrm{f}}(v)} m(\lambda) \lambda$, where $m: \sigma_{\mathrm{f}}(v) \rightarrow \mathbb{Z}$ has finite support, that is, $m(\lambda) \neq 0$ for at most finitely many $\lambda \in \sigma_{\mathrm{f}}(v)$. It is clear that $\bmod (v)$ carries the structure of a $\mathbb{Z}$-module and is the smallest additive subgroup of $\mathbb{R}$ containing $\sigma_{\mathrm{f}}(v)$.

We recall another concept of almost periodicity which is weaker than that of Bohr. Let $v \in L_{\text {loc }}^{q}(R, W)$, where $1 \leq q<\infty$ and $\varepsilon>0$. We say that $\tau \in R$ is an $\varepsilon$-period of $v$ (in the sense of Stepanov) if

$$
\left\|\left(\mathbf{S}_{\tau}-I\right) v\right\|_{S^{q}}=\sup _{a \in R}\left(\int_{a}^{a+1}\|v(s+\tau)-v(s)\|^{q} d s\right)^{1 / q} \leq \varepsilon .
$$

The set of $\varepsilon$-periods of $v$ (in the sense of Stepanov) is denoted by $P_{q}(v, \varepsilon)$. We say that $v$ is almost periodic in the sense of Stepanov if, for every $\varepsilon>0$, the set $P_{q}(v, \varepsilon)$ is relatively dense in $R$. The set of all functions in $L_{\text {loc }}^{q}(R, W)$ which are almost periodic in the sense of Stepanov is denoted by $S^{q}(R, W)$. It is clear that $A P(R, W) \subset S^{q}(R, W)$ (where the inclusion is strict), and, for every $v \in A P(R, W)$ and every $\varepsilon>0, P(v, \varepsilon) \subset P_{q}(v, \varepsilon)$. It is a routine exercise to prove that $S^{q}(R, W)$ is a closed subspace of $U L_{\text {loc }}^{q}(R, W)$ with respect to the Stepanov norm $\|\cdot\|_{S^{q}}$. It follows from (2.1) that, for $1 \leq p \leq q<\infty$ and $v \in L_{\mathrm{loc}}^{q}(R, W), P_{q}(v, \varepsilon) \subset P_{p}(\tau, \varepsilon)$, and consequently, $S^{q}(R, W) \subset S^{p}(R, W)$ (and the embedding is continuous).

Sometimes it will be convenient to associate with a function $v \in L_{\mathrm{loc}}^{q}(R, W)$ another function $\tilde{v}: R \rightarrow L^{q}([0,1], W)$ defined by

$$
(\tilde{v}(t))(s):=v(t+s) \quad \forall t \in R, \forall s \in[0,1]
$$

the so-called Bochner transform of $v$. Then, $\tilde{v} \in C\left(R, L^{q}([0,1], W)\right)$, and

$$
\|v\|_{S^{q}}=\|\tilde{v}\|_{\infty} \quad \forall v \in U L_{\mathrm{loc}}^{q}(R, W)
$$

that is, the Bochner transform restricted to $U L_{\text {loc }}^{q}(R, W)$ is an isometry. Furthermore, a function $v \in U L_{\mathrm{loc}}^{q}(R, W)$ is in $S^{q}(R, W)$ if, and only if, $\tilde{v} \in A P\left(R, L^{q}([0,1], W)\right)$. 
We remark that the Bochner transform is far from being surjective. In particular, there are elements in $A P\left(R, L^{q}([0,1], W)\right)$ which are not Bochner transforms of any function in $L_{\text {loc }}^{q}(R, W) .^{2}$

The following simple lemma is a consequence of Lemma 4.1 and (4.2).

Lemma 4.3 If $v \in S^{q}\left(\mathbb{R}_{+}, W\right)$, then, for every $\tau \in \mathbb{R}_{+},\left\|\mathbf{S}_{\tau} v\right\|_{S^{q}}=\|v\|_{S^{q}}$.

The space $A S^{q}\left(\mathbb{R}_{+}, W\right)$ of asymptotically almost periodic functions in the sense of Stepanov is defined as follows

$$
A S^{q}\left(\mathbb{R}_{+}, W\right):=S^{q}\left(\mathbb{R}_{+}, W\right)+U_{0} L_{\text {loc }}^{q}\left(\mathbb{R}_{+}, W\right) .
$$

Obviously, $A A P\left(\mathbb{R}_{+}, W\right) \subset A S^{q}\left(\mathbb{R}_{+}, W\right)$ and $A S^{q}\left(\mathbb{R}_{+}, W\right) \subset A S^{p}\left(\mathbb{R}_{+}, W\right)$ for all $1 \leq p \leq q<\infty$, and in both cases, the canonical injection is continuous. Noting that

$$
v \in U_{0} L_{\mathrm{loc}}^{q}\left(\mathbb{R}_{+}, W\right) \Leftrightarrow \tilde{v} \in C_{0}\left(\mathbb{R}_{+}, L^{q}([0,1], W)\right) \quad \forall v \in U L_{\mathrm{loc}}^{q}\left(\mathbb{R}_{+}, W\right),
$$

and

$$
\begin{aligned}
& v \in A S^{q}\left(\mathbb{R}_{+}, W\right) \Leftrightarrow \tilde{v} \in A A P\left(\mathbb{R}_{+}, L^{q}([0,1], W)\right) \\
& \forall v \in U L_{\text {loc }}^{q}\left(\mathbb{R}_{+}, W\right),
\end{aligned}
$$

it follows from (4.1) and (4.2) that

$$
\begin{gathered}
\|v+w\|_{S^{q}} \geq\|v\|_{S^{q}} \quad \forall v \in S^{q}\left(\mathbb{R}_{+}, W\right), \\
\forall w \in U_{0} L_{\mathrm{loc}}^{q}\left(\mathbb{R}_{+}, W\right) .
\end{gathered}
$$

It is an easy consequence of this inequality that $A S^{q}\left(\mathbb{R}_{+}, W\right)$ is a closed subspace of $U L_{\text {loc }}^{q}\left(\mathbb{R}_{+}, W\right)$. Moreover, (4.3) and (4.4) together with Lemma 4.2 and (4.2) yield the following result.

Lemma 4.4 Let $1 \leq q<\infty$. If $v \in A S^{q}\left(\mathbb{R}_{+}, W\right)$, then the decomposition $v=v^{\mathrm{s}}+w$, where $v^{\mathrm{s}} \in S^{q}\left(\mathbb{R}_{+}, W\right)$ and $w \in U_{0} L_{\mathrm{loc}}^{q}\left(\mathbb{R}_{+}, W\right)$, is unique.

In the following, for $v \in A S^{q}\left(\mathbb{R}_{+}, W\right)$, we let $v^{\text {s }}$ denote the unique function in $S^{q}\left(\mathbb{R}_{+}, W\right)$ such that $v-v^{\mathrm{s}} \in U_{0} L_{\text {loc }}^{q}\left(\mathbb{R}_{+}, W\right)$.

Let $v \in S^{q}\left(\mathbb{R}_{+}, W\right)$ and let $\tau_{k} \in P_{q}(v, 1 / k)$ for all $k \in \mathbb{N}$ and $\tau_{k} \rightarrow \infty$ as $k \rightarrow \infty$. Then, it can be shown that, for each $\tau>0,\left(v\left(\cdot+\tau_{k}\right)\right)_{k}$ is a Cauchy sequence in $L^{q}([-\tau, \tau], W)$ and hence defines a function $v_{\mathrm{e}} \in L_{\text {loc }}^{q}(\mathbb{R}, W)$. A straightforward argument shows that $\left.v_{\mathrm{e}}\right|_{\mathbb{R}_{+}}=v$ (i.e. $v_{\mathrm{e}}$ extends $v$ to $\left.\mathbb{R}\right), v_{\mathrm{e}} \in S^{q}(\mathbb{R}, W), P_{q}\left(v_{\mathrm{e}}, \varepsilon\right)=$ $\left\{ \pm \tau: \tau \in P_{q}(v, \varepsilon)\right\}$ for every $\varepsilon>0$, and the map $S^{q}\left(\mathbb{R}_{+}, W\right) \mapsto S^{q}(\mathbb{R}, W), v \mapsto v_{\mathrm{e}}$ is an isometric isomorphism.

We are now in the position to state and prove the main result of this paper.

2 Consider the constant function $F \in A P\left(R, L^{q}([0,1], W)\right)$ given by $F(t)=\lambda$, where $\lambda \in L^{q}([0,1], W)$ is such that $\left.\lambda\right|_{[0,1 / 2]}=0$ and $\left.\lambda\right|_{[1 / 2,1]} \neq 0$. Seeking a contradiction, suppose that there exists $f \in$ $L_{\text {loc }}^{q}(R, W)$ with $\tilde{f}=F$. Then, $f(t+s)=0$ for every $t \in R$ and almost every $s \in[0,1 / 2]$, implying that $f=0$, and thus, $F=\tilde{f}=0$ which is absurd. 
Theorem 4.5 Let $\Sigma=(\mathbb{T}, \Phi, \Psi, \mathbb{G})$ be a well-posed linear system, let $i, j \in$ $\{1,2\}, \quad K^{i j} \in \mathcal{L}\left(Y^{j}, U^{i}\right)$ and let $v^{*} \in S^{2}\left(\mathbb{R}_{+}, U\right)$. Assume that $\Sigma^{j i}=$ $\left(\mathbb{T}, \Phi E^{i}, P^{j} \Psi, P^{j} \mathbb{G} E^{i}\right)$ is optimizable and estimatable and $K^{i j} \in \mathbb{S}\left(P^{j} \mathbf{G} E^{i}\right)$. If $f: Y^{j} \rightarrow U^{i}$ satisfies (3.8) with $Z_{1}=Z_{2}=Y^{j}$, then there exists a unique pair $\left(x^{*}, y^{*}\right) \in A P\left(\mathbb{R}_{+}, X\right) \times S^{2}\left(\mathbb{R}_{+}, Y\right)$ such that $\left(v^{*}, x^{*}, y^{*}\right) \in \mathcal{B}$, for every $\varepsilon>0$, there exists $\delta>0$ such that $P_{2}\left(v^{*}, \delta\right) \subset P\left(x^{*}, \varepsilon\right) \cap P_{2}\left(y^{*}, \varepsilon\right)$ and the following statements hold.

(1) If $(v, x, y) \in \mathcal{B}$ is such that $v \in A S^{2}\left(\mathbb{R}_{+}, U\right)$ with $v^{\mathrm{s}}=v^{*}$, then

$\lim _{t \rightarrow \infty}\left(x(t)-x^{*}(t)\right)=0, \quad y \in U L_{\mathrm{loc}}^{2}\left(\mathbb{R}_{+}, Y\right)$ and $\left\|\mathbf{S}_{t}\left(y-y^{*}\right)\right\|_{S^{2}} \rightarrow 0$ as $t \rightarrow \infty$

that is, $x \in A A P\left(\mathbb{R}_{+}, X\right)$ with $x^{\text {ap }}=x^{*}$ and $y \in A S^{2}\left(\mathbb{R}_{+}, Y\right)$ with $y^{\mathrm{s}}=y^{*}$.

(2) If $(v, x, y) \in \mathcal{B}$ is such that $v-v^{*} \in U_{\alpha} L_{\mathrm{loc}}^{2}\left(\mathbb{R}_{+}, U\right)$ for some $\alpha>0$, then $\left(x(t)-x^{*}(t)\right) \rightarrow 0$ and $\left\|\mathbf{S}_{t}\left(y-y^{*}\right)\right\|_{S^{2}} \rightarrow 0$ exponentially fast as $t \rightarrow \infty$.

(3) If $v^{*}$ is periodic with period $\tau$, then $\left(x^{*}, y^{*}\right)$ is $\tau$-periodic.

(4) $\left(v_{\mathrm{e}}^{*}, x_{\mathrm{e}}^{*}, y_{\mathrm{e}}^{*}\right) \in \mathcal{B B}$ and there is no other pair $(\hat{x}, \hat{y}) \in B C(\mathbb{R}, X) \times L_{\text {loc }}^{2}(\mathbb{R}, Y)$ such that the triple $\left(v_{\mathrm{e}}^{*}, \hat{x}, \hat{y}\right)$ is in $\mathcal{B B}$.

(5) $\bmod \left(\widetilde{v_{\mathrm{e}}^{*}}\right) \supset \bmod \left(x_{\mathrm{e}}^{*}\right) \cup \bmod \left(\tilde{y}_{\mathrm{e}}^{*}\right)$.

Before proving Theorem 4.5, we provide some commentary.

Remark 4.6 (a) With regard to statement (1), note that if $v-v^{*} \in L^{2}\left(\mathbb{R}_{+}, U\right)$ or if $\left\|v-v^{*}\right\|_{L^{\infty}(t, \infty)} \rightarrow 0$ as $t \rightarrow \infty$, then $v \in A S^{2}\left(\mathbb{R}_{+}, U\right)$ with $v^{\mathrm{s}}=v^{*}$.

(b) In statement (3), $\tau$-periodicity of the $L_{\mathrm{loc}}^{2}$-function $y^{*}$ means that $y^{*}(t+\tau)=$ $y^{*}(t)$ for almost every $t \geq 0$.

(c) Statement (3) is not new. It is a consequence of [27, Theorem 4.4] which provides a certain extension of the centre manifold theorem for infinite-dimensional Lur'e systems driven by exosystems of a very general nature. Furthermore, statement (3) appears also in [16, Theorem 5.4] where it is derived using incremental ISS ideas. In the proof below, the result is recovered as a special case of statement (1).

(d) Let $v<0$ be the growth constant of the linear feedback system $\Sigma^{K}$ with $K$ given by (2.4), and let $v<\alpha<0$. It can be shown that $\left(x_{\mathrm{e}}^{*}, y_{\mathrm{e}}^{*}\right)$ is the unique element in $C(\mathbb{R}, X) \times L_{\text {loc }}^{2}(\mathbb{R}, Y)$ such that $\int_{\infty}^{0} e^{-2 \alpha t}\left\|y_{\mathrm{e}}^{*}(t)\right\|^{2} d t<\infty$ and $e^{-\alpha t} x_{\mathrm{e}}^{*}(t) \rightarrow 0$ as $t \rightarrow-\infty$ and which is a solution of the bilateral extension of (3.1) in the sense of [27] (see also [40, Sect. 5]).

(e) Note that if $v^{*} \in A P\left(\mathbb{R}_{+}, U\right)$ and $v \in A A P\left(\mathbb{R}_{+}, U\right)$ with $v^{\text {ap }}=v^{*}$, then $v^{*} \in S^{2}\left(\mathbb{R}_{+}, U\right)$ and $v \in A S^{2}\left(\mathbb{R}_{+}, U\right)$ with $v^{\mathrm{s}}=v^{\text {ap }}=v^{*}$, and Theorem 4.5 applies. Furthermore, in this case, it can be shown that $\sigma_{\mathrm{f}}\left(v_{\mathrm{e}}^{*}\right)=\sigma_{\sigma_{\mathrm{f}}}\left(\widetilde{v_{\mathrm{e}}^{*}}\right)$, and so, statement (5) can be written in the form $\bmod \left(v_{\mathrm{e}}^{*}\right) \supset \bmod \left(x_{\mathrm{e}}^{*}\right) \cup \bmod \left(\tilde{y}_{\mathrm{e}}^{*}\right)$. Of course, the extra regularity in the forcing provided by assuming that $v^{*} \in A P\left(\mathbb{R}_{+}, U\right)$ and $v \in A A P\left(\mathbb{R}_{+}, U\right)$ is not sufficient to guarantee that $y^{*} \in A P\left(\mathbb{R}_{+}, Y\right)$ and/or $y \in$ $A A P\left(\mathbb{R}_{+}, Y\right)$.

Proof of Theorem 4.5 Let $v^{*} \in S^{2}\left(\mathbb{R}_{+}, U\right)$. It follows from (3.8), Proposition 3.1 and Lemma 3.2 (with $K=E^{i} K^{i j} P^{j}$ ) that there exists a pair $(x, y) \in C\left(\mathbb{R}_{+}, X\right) \times$ 
$L_{\text {loc }}^{2}\left(\mathbb{R}_{+}, Y\right)$ such that $\left(v^{*}, x, y\right) \in \mathcal{B}$. Let $\left(v^{\mathrm{eq}}, x^{\mathrm{eq}}, y^{\mathrm{eq}}\right)$ be an arbitrary equilibrium triple of (3.1) (the existence of which is guaranteed by Proposition 3.3). Setting $r:=1 /\left\|\left(P^{j} \mathbf{G} E^{i}\right)^{K^{i j}}\right\|_{H^{\infty}}$, it follows from Lemma 2.1 that $\mathbb{B}\left(K^{i j}, r\right) \subset \mathbb{S}\left(P^{j} \mathbf{G} E^{i}\right)$, and hence, the hypotheses of Theorem 3.4 are satisfied. Therefore, applying statements (3) and (4) of Theorem 3.4 with $\left(v_{1}, x_{1}, y_{1}\right)=\left(v^{*}, x, y\right)$ and $\left(v_{2}, x_{2}, y_{2}\right)=$ $\left(v^{\mathrm{eq}} \vartheta, x^{\mathrm{eq}} \vartheta, y^{\mathrm{eq}} \vartheta\right)$, where $\vartheta$ is the constant function $\vartheta(t) \equiv 1$, it follows that $x$ is bounded, and so $x \in B C\left(\mathbb{R}_{+}, X\right)$, and, furthermore, $y \in U L_{\text {loc }}^{2}\left(\mathbb{R}_{+}, Y\right)$. We set

$$
\rho:=2\|x\|_{\infty},
$$

and choose a non-decreasing sequence $\left(\tau_{k}\right)_{k \in \mathbb{N}}$ such that

$$
\tau_{k} \in P_{2}\left(v^{*}, 1 / k^{2}\right) \text { and } \tau_{k}>k \quad \forall k \in \mathbb{N} \text {. }
$$

We proceed in several steps.

Step 1: Construction of $x^{*}$. We are going to show that $\left(\mathbf{S}_{\tau_{k}} x\right)_{k}$ is a Cauchy sequence in $B C\left(\mathbb{R}_{+}, X\right){ }^{3}$ To this end, we note that

$$
\begin{aligned}
\left(\int_{a}^{a+k}\left\|v^{*}\left(t+\tau_{k}\right)-v^{*}(t)\right\|^{2} d t\right)^{1 / 2} & \leq \sum_{j=1}^{k}\left(\int_{a+j-1}^{a+j}\left\|v^{*}\left(t+\tau_{k}\right)-v^{*}(t)\right\|^{2} d t\right)^{1 / 2} \\
& \leq \frac{1}{k} \quad \forall a \geq 0, \quad \forall k \in \mathbb{N} .
\end{aligned}
$$

Consequently,

$$
\sup _{a \geq 0}\left(\int_{a}^{a+k}\left\|v^{*}\left(t+\tau_{k}\right)-v^{*}(t)\right\|^{2}\right)^{1 / 2} \leq \frac{1}{k} .
$$

Since $\left(\mathbf{S}_{\tau} v^{*}, \mathbf{S}_{\tau} x, \mathbf{S}_{\tau} y\right) \in \mathcal{B}$ for all $\tau \geq 0$, it follows from statement (1) of Theorem 3.4 that there exist constants $\Gamma_{2}, \gamma>0$ such that

$$
\begin{aligned}
\left\|\left(\mathbf{S}_{\sigma} x\right)(s)-\left(\mathbf{S}_{\sigma+\tau} x\right)(s)\right\| \leq & \Gamma_{2}\left(\rho e^{-\gamma\left(s-s_{0}\right)}\right. \\
& \left.+\left\|\mathbf{S}_{\sigma} v^{*}-\mathbf{S}_{\sigma+\tau} v^{*}\right\|_{L^{2}\left(s_{0}, s\right)}\right) \forall\left(s, s_{0}\right) \in \Delta, \forall \sigma, \tau \geq 0 .
\end{aligned}
$$

Trivially, for $k, \ell \in \mathbb{N}$ with $k \geq \ell$,

$$
\left(\mathbf{S}_{\tau_{\ell}} x\right)(t)-\left(\mathbf{S}_{\tau_{k}} x\right)(t)=\left(\mathbf{S}_{t} x\right)\left(\tau_{\ell}\right)-\left(\mathbf{S}_{t+\tau_{k}-\tau_{\ell}} x\right)\left(\tau_{\ell}\right), \quad \forall t \geq 0,
$$

and so, setting

$$
I(t ; k, \ell):=\left\|\mathbf{S}_{t} v^{*}-\mathbf{S}_{t+\tau_{k}-\tau_{\ell}} v^{*}\right\|_{L^{2}\left(\tau_{\ell}-\ell, \tau_{\ell}\right)} \quad \forall t \geq 0
$$

\footnotetext{
3 Thereby extending an idea from [2] where a similar argument is used to establish the existence of a periodic solution of periodically forced finite-dimensional systems.
} 
and invoking (4.6) with $s=\tau_{\ell}, s_{0}=\tau_{\ell}-\ell, \sigma=t$ and $\tau=\tau_{k}-\tau_{\ell}$, we arrive at

$$
\left\|\left(\mathbf{S}_{\tau_{\ell}} x\right)(t)-\left(\mathbf{S}_{\tau_{k}} x\right)(t)\right\| \leq \Gamma_{2}\left(\rho e^{-\gamma \ell}+I(t ; k, \ell)\right) \quad \forall t \geq 0, \forall k, \ell \in \mathbb{N} \text { s.t. } k \geq \ell .
$$

Now

$$
I(t ; k, \ell) \leq\left\|\mathbf{S}_{t} v^{*}-\mathbf{S}_{t+\tau_{k}} v^{*}\right\|_{L^{2}\left(\tau_{\ell}-\ell, \tau_{\ell}\right)}+\left\|\mathbf{S}_{t+\tau_{k}} v^{*}-\mathbf{S}_{t+\tau_{k}-\tau_{\ell}} v^{*}\right\|_{L^{2}\left(\tau_{\ell}-\ell, \tau_{\ell}\right)},
$$

and so, changing variables in the two terms on the right-hand side, we obtain that, for all $t \geq 0$ and all $k, \ell \in \mathbb{N}$ such that $k \geq \ell$,

$$
\begin{aligned}
I(t ; k, \ell) & \leq\left\|v^{*}-\mathbf{S}_{\tau_{k}} v^{*}\right\|_{L^{2}\left(t+\tau_{\ell}-\ell, t+\tau_{\ell}\right)}+\left\|\mathbf{S}_{\tau_{\ell}} v^{*}-v^{*}\right\|_{L^{2}\left(t+\tau_{k}-\ell, t+\tau_{k}\right)} \\
& \leq\left\|v^{*}-\mathbf{S}_{\tau_{k}} v^{*}\right\|_{L^{2}\left(t+\tau_{\ell}-\ell, t+\tau_{\ell}-\ell+k\right)}+\left\|\mathbf{S}_{\tau_{\ell}} v^{*}-v^{*}\right\|_{L^{2}\left(t+\tau_{k}-\ell, t+\tau_{k}\right)} .
\end{aligned}
$$

Consequently, by (4.5),

$$
I(t ; k, \ell) \leq \frac{1}{k}+\frac{1}{\ell} \quad \forall t \geq 0, \quad \forall k, \ell \in \mathbb{N} \text { s.t. } k \geq \ell
$$

and it follows from (4.7) that

$$
\left\|\left(\mathbf{S}_{\tau_{\ell}} x\right)(t)-\left(\mathbf{S}_{\tau_{k}} x\right)(t)\right\| \leq \Gamma_{2}\left(\rho e^{-\gamma \ell}+1 / k+1 / \ell\right) \quad \forall t \geq 0, \quad \forall k, \ell \in \mathbb{N} \text { s.t. } k \geq \ell
$$

This shows that $\left(\mathbf{S}_{\tau_{k}} x\right)_{k}$ is a Cauchy sequence in $B C\left(\mathbb{R}_{+}, X\right)$, the limit of which we denote by $x^{*}$.

To show that $x^{*} \in A P\left(\mathbb{R}_{+}, X\right)$, let $\varepsilon>0$, choose $k_{\varepsilon} \in \mathbb{N}$ such that $\rho e^{-\gamma k_{\varepsilon}} \leq$ $\varepsilon /\left(2 \Gamma_{2}\right)$ and set

$$
\eta_{\varepsilon}:=\frac{\varepsilon}{2 k_{\varepsilon} \Gamma_{2}}
$$

Let $\tau \in P_{2}\left(v^{*}, \eta_{\varepsilon}\right)$. We will show that $P_{2}\left(v^{*}, \eta_{\varepsilon}\right) \subset P\left(x^{*}, \varepsilon\right)$. Obviously,

$$
\left(\mathbf{S}_{\tau_{k}} x\right)(t+\tau)-\left(\mathbf{S}_{\tau_{k}} x\right)(t)=\left(\mathbf{S}_{t+\tau} x\right)\left(\tau_{k}\right)-\left(\mathbf{S}_{t} x\right)\left(\tau_{k}\right) \quad \forall t \geq 0, \forall k \in \mathbb{N}
$$

and so, by (4.6) with $s=\tau_{k}, \sigma=t$ and $s_{0}=\tau_{k}-k_{\varepsilon}$,

$$
\begin{aligned}
& \left\|\left(\mathbf{S}_{\tau_{k}} x\right)(t+\tau)-\left(\mathbf{S}_{\tau_{k}} x\right)(t)\right\| \\
& \quad \leq \Gamma_{2}\left(\rho e^{-\gamma k_{\varepsilon}}+\left\|\mathbf{S}_{t} v^{*}-\mathbf{S}_{t+\tau} v^{*}\right\|_{L^{2}\left(\tau_{k}-k_{\varepsilon}, \tau_{k}\right)}\right) \quad \forall t \geq 0, \quad \forall k \geq k_{\varepsilon} .
\end{aligned}
$$

Now

$$
\begin{aligned}
& \left\|\mathbf{S}_{t} v^{*}-\mathbf{S}_{t+\tau} v^{*}\right\|_{L^{2}\left(\tau_{k}-k_{\varepsilon}, \tau_{k}\right)} \\
& \quad=\left\|v^{*}-\mathbf{S}_{\tau} v^{*}\right\|_{L^{2}\left(t+\tau_{k}-k_{\varepsilon}, t+\tau_{k}\right)} \leq k_{\varepsilon} \eta_{\varepsilon}=\frac{\varepsilon}{2 \Gamma_{2}} \quad \forall t \geq 0, \quad \forall k \geq k_{\varepsilon},
\end{aligned}
$$


and thus,

$$
\left\|\left(\mathbf{S}_{\tau_{k}} x\right)(t+\tau)-\left(\mathbf{S}_{\tau_{k}} x\right)(t)\right\| \leq \frac{\varepsilon}{2}+\frac{\varepsilon}{2}=\varepsilon \quad \forall t \geq 0, \quad \forall k \geq k_{\varepsilon} .
$$

Letting $k \rightarrow \infty$, we obtain

$$
\left\|x^{*}(t+\tau)-x^{*}(t)\right\| \leq \varepsilon \quad \forall t \geq 0,
$$

establishing that $P_{2}\left(v^{*}, \eta_{\varepsilon}\right) \subset P\left(x^{*}, \varepsilon\right)$. The set $P_{2}\left(v^{*}, \eta_{\varepsilon}\right)$ is relatively dense in $\mathbb{R}_{+}$, and, a fortiori, $P\left(x^{*}, \varepsilon\right)$ is also relatively dense in $\mathbb{R}_{+}$. Since $\varepsilon$ was arbitrary, we conclude that $x^{*} \in A P\left(\mathbb{R}_{+}, X\right)$.

Step 2: Construction of $y^{*}$. By statement (4) of Theorem 3.4 there exists a constant $\tilde{\Gamma}>0$ such that

$$
\begin{aligned}
\left\|\mathbf{S}_{t+\tau_{k}} y-\mathbf{S}_{\tau_{\ell}} y\right\|_{S^{2}} \leq & \tilde{\Gamma}\left(\left\|\left(\mathbf{S}_{t+\tau_{k}} x\right)(0)-\left(\mathbf{S}_{\tau_{\ell}} x\right)(0)\right\|\right. \\
& \left.+\left\|\mathbf{S}_{t+\tau_{k}} v^{*}-\mathbf{S}_{\tau_{\ell}} v^{*}\right\|_{S^{2}}\right) \quad \forall t \geq 0, \forall k, \ell \in \mathbb{N} .
\end{aligned}
$$

Obviously, $\mathbf{S}_{\tau_{k}} v^{*} \rightarrow v^{*}$ in $S^{2}\left(\mathbb{R}_{+}, U\right)$ and $\left(\mathbf{S}_{\tau_{k}} x\right)(0) \rightarrow x^{*}(0)$ as $k \rightarrow \infty$, and so it follows from (4.8) with $t=0$ that $\left(\mathbf{S}_{\tau_{k}} y\right)_{k}$ is a Cauchy sequence in $U L_{\text {loc }}^{2}\left(\mathbb{R}_{+}, Y\right)$, the limit of which we denote by $y^{*}$. Letting $k \rightarrow \infty$ and $\ell \rightarrow \infty$ in (4.8) we arrive at

$$
\left\|\mathbf{S}_{t} y^{*}-y^{*}\right\|_{S^{2}} \leq \tilde{\Gamma}\left(\left\|\left(\mathbf{S}_{t} x^{*}\right)(0)-x^{*}(0)\right\|+\left\|\mathbf{S}_{t} v^{*}-v^{*}\right\|_{S^{2}}\right) \quad \forall t \geq 0 .
$$

Now let $\varepsilon>0$, set $\tilde{\varepsilon}:=\varepsilon /(2 \tilde{\Gamma})$ and

$$
\delta_{\varepsilon}:=\min \left\{\eta_{\varepsilon}, \eta_{\tilde{\varepsilon}}, \tilde{\varepsilon}\right\}
$$

and let $\tau \in P_{2}\left(v^{*}, \delta_{\varepsilon}\right)$. Then, $\tau \in P_{2}\left(v^{*}, \eta_{\tilde{\varepsilon}}\right)$, and consequently, by what we proved in Step $1, \tau \in P\left(x^{*}, \tilde{\varepsilon}\right)$. An application of (4.9) with $t=\tau$ yields

$$
\left\|\mathbf{S}_{\tau} y^{*}-y^{*}\right\|_{S^{2}} \leq \tilde{\Gamma}\left(\tilde{\varepsilon}+\delta_{\varepsilon}\right) \leq \varepsilon
$$

Hence, $P_{2}\left(v^{*}, \delta_{\varepsilon}\right) \subset P_{2}\left(y^{*}, \varepsilon\right)$. Therefore, the relative denseness of $P_{2}\left(v^{*}, \delta_{\varepsilon}\right)$ implies that of $P_{2}\left(y^{*}, \varepsilon\right)$, showing that $y^{*} \in S^{2}\left(\mathbb{R}_{+}, Y\right)$. By the definition of $\delta_{\varepsilon}$, we have that $P_{2}\left(v^{*}, \delta_{\varepsilon}\right) \subset P_{2}\left(v^{*}, \eta_{\varepsilon}\right)$, and so, by Step $1, P_{2}\left(v^{*}, \delta_{\varepsilon}\right) \subset P\left(x^{*}, \varepsilon\right)$. Consequently, $P_{2}\left(v^{*}, \delta_{\varepsilon}\right) \subset P\left(x^{*}, \varepsilon\right) \cap P_{2}\left(y^{*}, \varepsilon\right)$.

Step 3: $\left(v^{*}, x^{*}, y^{*}\right) \in \mathcal{B}$ and uniqueness of $\left(x^{*}, y^{*}\right)$ within $A P\left(\mathbb{R}_{+}, X\right) \times$ $S^{2}\left(\mathbb{R}_{+}, Y\right)$. Since $\left(\mathbf{S}_{\tau_{k}} v^{*}, \mathbf{S}_{\tau_{k}} x, \mathbf{S}_{\tau_{k}} y\right)$ is in $\mathcal{B}$ for all $k \in \mathbb{N},\left\|\mathbf{S}_{\tau_{k}} v^{*}-v^{*}\right\|_{S^{2}} \rightarrow 0$, $\left\|\mathbf{S}_{\tau_{k}} y-y^{*}\right\|_{S^{2}} \rightarrow 0$ and $\left\|\mathbf{S}_{\tau_{k}} x-x^{*}\right\|_{\infty} \rightarrow 0$ as $k \rightarrow \infty$, it follows from (3.1), the continuity properties of well-posed linear systems and the global Lipschitz property of $f$ that $\left(v^{*}, x^{*}, y^{*}\right) \in \mathcal{B}$.

To prove uniqueness of $\left(x^{*}, y^{*}\right)$, assume that $\left(x^{\sharp}, y^{\sharp}\right) \in A P\left(\mathbb{R}_{+}, X\right) \times S^{2}\left(\mathbb{R}_{+}, Y\right)$ is such that $\left(v^{*}, x^{\sharp}, y^{\sharp}\right) \in \mathcal{B}$. Then, appealing to statement (1) of Theorem 3.4, we see that $x^{*}(t)-x^{\sharp}(t) \rightarrow 0$ as $t \rightarrow \infty$. But the function $x^{*}-x^{\sharp}$ is in $A P\left(\mathbb{R}_{+}, X\right)$, and so, 
invoking Lemma 4.1, we conclude that $x^{*}=x^{\sharp}$. Statement (2) of Theorem 3.4 now implies that $y^{*}=y^{\sharp}$.

Step 4: Proof of statements (1)-(3). Let $(v, x, y) \in \mathcal{B}$ be such that $v \in A S^{2}\left(\mathbb{R}_{+}, U\right)$ with $v^{\mathrm{s}}=v^{*}$. An application of statements (3) and (4) of Theorem 3.4 to the trajectories $\left(v_{1}, x_{1}, y_{1}\right)=(v, x, y)$ and $\left(v_{2}, x_{2}, y_{2}\right)=\left(v^{*}, x^{*}, y^{*}\right)$ shows that statements $(1)$ and (2) hold.

$\tilde{\Gamma}_{2}>0$ and $\tilde{\gamma}>0$ such that

$$
\left\|x(t)-x^{*}(t)\right\| \leq \tilde{\Gamma}_{2}\left(e^{-\tilde{\gamma}\left(t-t_{0}\right)}\left\|x\left(t_{0}\right)-x^{*}\left(t_{0}\right)\right\|+\left\|\mathbf{S}_{t_{0}}\left(v-v^{*}\right)\right\|_{S^{2}}\right) \quad \forall\left(t, t_{0}\right) \in \Delta
$$

To prove statement (3), assume that $v^{*}$ is $\tau$-periodic for some $\tau>0$. Then, $\tau \in$ $P_{2}\left(v^{*}, \delta\right)$ for every $\delta>0$ and so, $\tau \in P\left(x^{*}, \varepsilon\right) \cap P_{2}\left(y^{*}, \varepsilon\right)$ for every $\varepsilon>0$, implying that $x^{*}$ and $y^{*}$ are $\tau$-periodic.

Step 5: Proof of statement (4). To show that $\left(v_{\mathrm{e}}^{*}, x_{\mathrm{e}}^{*}, y_{\mathrm{e}}^{*}\right) \in \mathcal{B B}$, we choose $\delta_{k}>0$ such that

$$
P_{2}\left(v^{*}, \delta_{k}\right) \subset P\left(x^{*}, 1 / k\right) \cap P_{2}\left(y^{*}, 1 / k\right) \quad \forall k \in \mathbb{N} .
$$

The existence of such numbers $\delta_{k}$ is guaranteed by Steps 1 and 2. Setting $\eta_{k}:=$ $\min \left(\delta_{k}, 1 / k\right)$, we have that $\eta_{k} \rightarrow 0$ as $k \rightarrow \infty$ and

$$
P_{2}\left(v^{*}, \eta_{k}\right) \subset P\left(x^{*}, 1 / k\right) \cap P_{2}\left(y^{*}, 1 / k\right) \quad \forall k \in \mathbb{N} .
$$

Let $t_{0} \in \mathbb{R}$ and $\tau_{k} \in P_{2}\left(v^{*}, \eta_{k}\right)$ such that $\tau_{k} \geq \max \left(0,-t_{0}\right)$ for all $k \in \mathbb{N}$. The latter ensures that $t_{0}+\tau_{k} \geq 0$ for all $k \in \mathbb{N}$. Noting that

$$
\begin{aligned}
& x_{\mathrm{e}}^{*}\left(t+\tau_{k}\right)=x_{\mathrm{e}}^{*}\left(t-t_{0}+\tau_{k}+t_{0}\right)=x^{*}\left(t-t_{0}+\tau_{k}+t_{0}\right) \\
& \quad=\left(\mathbf{S}_{t_{0}+\tau_{k}} x^{*}\right)\left(t-t_{0}\right) \quad \forall t \geq t_{0}, \quad \forall k \in \mathbb{N},
\end{aligned}
$$

we conclude that

$$
\begin{aligned}
& x_{\mathrm{e}}^{*}\left(t+\tau_{k}\right)=\left(\mathbf{S}_{t_{0}+\tau_{k}} x^{*}\right)\left(t-t_{0}\right) \\
& \quad=\mathbb{T}_{t-t_{0}}\left(\mathbf{S}_{t_{0}+\tau_{k}} x^{*}\right)(0)+\Phi_{t-t_{0}} \mathbf{P}_{t-t_{0}} \mathbf{S}_{t_{0}+\tau_{k}} u^{*} \quad \forall t \geq t_{0}, \quad \forall k \in \mathbb{N},
\end{aligned}
$$

where $u^{*}:=E^{i}\left(f \circ P^{j} y^{*}\right)+v^{*}$. Since $v^{*} \in S^{2}\left(\mathbb{R}_{+}, U\right), y^{*} \in S^{2}\left(\mathbb{R}_{+}, Y\right)$ and $f$ is globally Lipschitz, it follows that $u^{*} \in S^{2}\left(\mathbb{R}_{+}, U\right)$. Trivially, by (4.10),

$$
x_{\mathrm{e}}^{*}\left(t+\tau_{k}\right)=\mathbb{T}_{t-t_{0}} x_{\mathrm{e}}^{*}\left(t_{0}+\tau_{k}\right)+\Phi_{t-t_{0}} \mathbf{P}_{t-t_{0}} \mathbf{S}_{t_{0}+\tau_{k}} u_{\mathrm{e}}^{*} \quad \forall t \geq t_{0}, \quad \forall k \in \mathbb{N} .
$$

Obviously, $u_{\mathrm{e}}^{*}=E^{i}\left(f \circ P^{j} y_{\mathrm{e}}^{*}\right)+v_{\mathrm{e}}^{*}$. As $\tau_{k} \in P_{2}\left(v_{\mathrm{e}}^{*}, \eta_{k}\right) \subset P\left(x_{\mathrm{e}}^{*}, 1 / k\right) \cap P_{2}\left(y_{\mathrm{e}}^{*}, 1 / k\right)$, we have 
$\left\|\mathbf{S}_{\tau_{k}} v_{\mathrm{e}}^{*}-v_{\mathrm{e}}^{*}\right\|_{S^{2}} \rightarrow 0, \quad\left\|\mathbf{S}_{\tau_{k}} x_{\mathrm{e}}^{*}-x_{\mathrm{e}}^{*}\right\|_{\infty} \rightarrow 0 \quad$ and $\left\|\mathbf{S}_{\tau_{k}} y_{\mathrm{e}}^{*}-y_{\mathrm{e}}^{*}\right\|_{S^{2}} \rightarrow 0 \quad$ as $k \rightarrow \infty$

which in turn implies that

$$
\left\|\mathbf{S}_{\tau_{k}} u_{\mathrm{e}}^{*}-u_{\mathrm{e}}^{*}\right\|_{S^{2}} \rightarrow 0 \text { as } k \rightarrow \infty
$$

Therefore, letting $k \rightarrow \infty$ in (4.11), we arrive at

$$
x_{\mathrm{e}}^{*}(t)=\mathbb{T}_{t-t_{0}} x_{\mathrm{e}}^{*}\left(t_{0}\right)+\Phi_{t-t_{0}} \mathbf{P}_{t-t_{0}} \mathbf{S}_{t_{0}} u_{\mathrm{e}}^{*} \forall t \geq t_{0} .
$$

Furthermore, on $\mathbb{R}_{+}, \mathbf{S}_{t_{0}+\tau_{k}} u_{\mathrm{e}}^{*}=\mathbf{S}_{t_{0}+\tau_{k}} u^{*}, \mathbf{S}_{t_{0}+\tau_{k}} x_{\mathrm{e}}^{*}=\mathbf{S}_{t_{0}+\tau_{k}} x^{*}$ and $\mathbf{S}_{t_{0}+\tau_{k}} y_{\mathrm{e}}^{*}=$ $\mathbf{S}_{t_{0}+\tau_{k}} y^{*}$, and thus, as

$$
\mathbf{P}_{t-t_{0}} \mathbf{S}_{t_{0}+\tau_{k}} y^{*}=\Psi_{t-t_{0}}\left(\mathbf{S}_{t_{0}+\tau_{k}} x^{*}\right)(0)+\mathbb{G}_{t-t_{0}} \mathbf{P}_{t-t_{0}} \mathbf{S}_{t_{0}+\tau_{k}} u^{*} \quad \forall t \geq t_{0},
$$

we obtain

$$
\mathbf{P}_{t-t_{0}} \mathbf{S}_{\tau_{k}} \mathbf{S}_{t_{0}} y_{\mathrm{e}}^{*}=\Psi_{t-t_{0}}\left(\mathbf{S}_{\tau_{k}} \mathbf{S}_{t_{0}} x_{\mathrm{e}}^{*}\right)(0)+\mathbb{G}_{t-t_{0}} \mathbf{P}_{t-t_{0}} \mathbf{S}_{\tau_{k}} \mathbf{S}_{t_{0}} u_{\mathrm{e}}^{*} \quad \forall t \geq t_{0}
$$

By (4.12) and (4.13),

$$
\begin{aligned}
& \left\|\mathbf{S}_{\tau_{k}} \mathbf{S}_{t_{0}} u_{\mathrm{e}}^{*}-\mathbf{S}_{t_{0}} u_{\mathrm{e}}^{*}\right\|_{S^{2}} \rightarrow 0, \quad\left\|\mathbf{S}_{\tau_{k}} \mathbf{S}_{t_{0}} x_{\mathrm{e}}^{*}-\mathbf{S}_{t_{0}} x_{\mathrm{e}}^{*}\right\|_{\infty} \rightarrow 0 \text { and } \\
& \left\|\mathbf{S}_{\tau_{k}} \mathbf{S}_{t_{0}} y_{\mathrm{e}}^{*}-\mathbf{S}_{t_{0}} y_{\mathrm{e}}^{*}\right\|_{S^{2}} \rightarrow 0 \quad \text { as } k \rightarrow \infty
\end{aligned}
$$

and thus, letting $k \rightarrow \infty$ in (4.15) leads to

$$
\mathbf{P}_{t-t_{0}} \mathbf{S}_{t_{0}} y_{\mathrm{e}}^{*}=\Psi_{t-t_{0}} x_{\mathrm{e}}^{*}\left(t_{0}\right)+\mathbb{G}_{t-t_{0}} \mathbf{P}_{t-t_{0}} \mathbf{S}_{t_{0}} u_{\mathrm{e}}^{*} \forall t \geq t_{0} .
$$

Since $t_{0} \in \mathbb{R}$ was arbitrary and $\mathbf{S}_{t_{0}} u_{\mathrm{e}}^{*}=E^{i}\left(f \circ P^{j} \mathbf{S}_{t_{0}} y_{\mathrm{e}}^{*}\right)+\mathbf{S}_{t_{0}} v_{\mathrm{e}}^{*}$, it follows from (4.14) and (4.16) that $\left(v_{\mathrm{e}}^{*}, x_{\mathrm{e}}^{*}, y_{\mathrm{e}}^{*}\right) \in \mathcal{B B}$.

To show that $\left(x_{\mathrm{e}}^{*}, y_{\mathrm{e}}^{*}\right)$ is the unique pair in $B C(\mathbb{R}, X) \times L_{\text {loc }}^{2}(\mathbb{R}, Y)$ satisfying $\left(v_{\mathrm{e}}^{*}, x_{\mathrm{e}}^{*}, y_{\mathrm{e}}^{*}\right) \in \mathcal{B B}$, let $(\hat{x}, \hat{y}) \in B C(\mathbb{R}, X) \times L_{\text {loc }}^{2}(\mathbb{R}, Y)$ be such that $\left(v_{\mathrm{e}}^{*}, \hat{x}, \hat{y}\right) \in \mathcal{B B}$. We have to show that $(\hat{x}, \hat{y})=\left(x_{\mathrm{e}}^{*}, y_{\mathrm{e}}^{*}\right)$. To this end, note that, for any $\sigma \in \mathbb{R}$, the restrictions of $\left(\mathbf{S}_{\sigma} v_{\mathrm{e}}^{*}, \mathbf{S}_{\sigma} x_{\mathrm{e}}^{*}, \mathbf{S}_{\sigma} y_{\mathrm{e}}^{*}\right)$ and $\left(\mathbf{S}_{\sigma} v_{\mathrm{e}}^{*}, \mathbf{S}_{\sigma} \hat{x}, \mathbf{S}_{\sigma} \hat{y}\right)$ to $\mathbb{R}_{+}$are in $\mathcal{B}$. Hence, by statement (1) of Theorem 3.4, there exist $\Gamma_{2}>0$ and $\gamma>0$ such that

$$
\left\|\left(\mathbf{S}_{\sigma} x_{\mathrm{e}}^{*}\right)(s)-\left(\mathbf{S}_{\sigma} \hat{x}\right)(s)\right\| \leq \Gamma_{2} e^{-\gamma s}\left\|x_{\mathrm{e}}^{*}(\sigma)-\hat{x}(\sigma)\right\| \quad \forall s \geq 0, \forall \sigma \in \mathbb{R} .
$$

Let $t \in \mathbb{R}$ and $\varepsilon>0$. Choose $\sigma \leq t$ such that

$$
\Gamma_{2} e^{-\gamma(t-\sigma)}\left\|x_{\mathrm{e}}^{*}-\hat{x}\right\|_{\infty} \leq \varepsilon
$$

An application of (4.17) wit $s=t-\sigma$ yields

$$
\left\|x_{\mathrm{e}}^{*}(t)-\hat{x}(t)\right\|=\left\|\left(\mathbf{S}_{\sigma} x_{\mathrm{e}}^{*}\right)(t-\sigma)-\left(\mathbf{S}_{\sigma} \hat{x}\right)(t-\sigma)\right\| \leq \Gamma_{2} e^{-\gamma(t-\sigma)}\left\|x_{\mathrm{e}}^{*}-\hat{x}\right\|_{\infty} \leq \varepsilon .
$$


Now $t \in \mathbb{R}$ and $\varepsilon>0$ were arbitrary, and consequently, $\hat{x}=x_{\mathrm{e}}^{*}$. An application of statement (4) of Theorem 3.4 (with $\left.t_{0}=0\right)$ ) to the restrictions of $\left(\mathbf{S}_{\sigma} v_{\mathrm{e}}^{*}, \mathbf{S}_{\sigma} x_{\mathrm{e}}^{*}, \mathbf{S}_{\sigma} y_{\mathrm{e}}^{*}\right)$ and $\left(\mathbf{S}_{\sigma} v_{\mathrm{e}}^{*}, \mathbf{S}_{\sigma} x_{\mathrm{e}}^{*}, \mathbf{S}_{\sigma} \hat{y}\right)$ to $\mathbb{R}_{+}$, where $\sigma \in \mathbb{R}$, shows that $\left(\mathbf{S}_{\sigma} \hat{y}\right)(t)=\left(\mathbf{S}_{\sigma} y_{\mathrm{e}}^{*}\right)(t)$ for almost every $t \geq 0$. Therefore, $\hat{y}(t)=y_{\mathrm{e}}^{*}(t)$ for almost every $t \geq \sigma$. Letting $\sigma \rightarrow-\infty$ yields that $\hat{y}=y_{\mathrm{e}}^{*}$.

Step 6: Proof of statement (5). Let $\left(\sigma_{k}\right)_{k}$ be a sequence in $\mathbb{R}$ such that $\left(\mathbf{S}_{\sigma_{k}} \widetilde{v_{\mathrm{e}}^{*}}\right)_{k}$ converges in $A P\left(\mathbb{R}, L^{1}([0,1), U)\right)$. By [1, Statement $\mathrm{X}$ on p. 34], it is sufficient to prove that the sequences $\left(\mathbf{S}_{\sigma_{k}} x_{\mathrm{e}}^{*}\right)_{k}$ and $\left(\mathbf{S}_{\sigma_{k}} \tilde{y}_{\mathrm{e}}^{*}\right)_{k}$ converge in $A P(\mathbb{R}, X)$ and $A P\left(\mathbb{R}, L^{1}([0,1), Y)\right)$, respectively. To this end, let $\varepsilon>0$ and set $r:=2\left\|x_{\mathrm{e}}^{*}\right\|_{\infty}=$ $2\left\|x^{*}\right\|_{\infty}$. Obviously, for each $k \in \mathbb{N}$, the restriction of $\left(\mathbf{S}_{\sigma_{k}} v_{\mathrm{e}}^{*}, \mathbf{S}_{\sigma_{k}} x_{\mathrm{e}}^{*}, \mathbf{S}_{\sigma_{k}} y_{\mathrm{e}}^{*}\right)$ to $\mathbb{R}_{+}$is in $\mathcal{B}$. Consequently, by statements (3) and (4) of Theorem 3.4,

$$
\left\|\left(\mathbf{S}_{\sigma_{k}} x_{\mathrm{e}}^{*}\right)(t)-\left(\mathbf{S}_{\sigma_{\ell}} x_{\mathrm{e}}^{*}\right)(t)\right\| \leq \tilde{\Gamma}_{2}\left(e^{-\tilde{\gamma} t} r+\left\|\mathbf{S}_{\sigma_{k}} v_{\mathrm{e}}^{*}-\mathbf{S}_{\sigma_{\ell}} v_{\mathrm{e}}^{*}\right\|_{S^{2}}\right) \quad \forall t \geq 0,
$$

and

$$
\left\|\mathbf{S}_{\sigma_{k}} y_{\mathrm{e}}^{*}-\mathbf{S}_{\sigma_{\ell}} y_{\mathrm{e}}^{*}\right\|_{S^{2}} \leq \tilde{\Gamma}\left(\left\|\left(\mathbf{S}_{\sigma_{k}} x_{\mathrm{e}}^{*}\right)(0)-\left(\mathbf{S}_{\sigma_{\ell}} x_{\mathrm{e}}^{*}\right)(0)\right\|+\left\|\mathbf{S}_{\sigma_{k}} v_{\mathrm{e}}^{*}-\mathbf{S}_{\sigma_{\ell}} v_{\mathrm{e}}^{*}\right\|_{S^{2}}\right)
$$

where $\tilde{\Gamma}_{2}, \tilde{\Gamma}$ and $\tilde{\gamma}$ are positive constants. Since $\left(\mathbf{S}_{\sigma_{k}} \widetilde{v}_{\mathrm{e}}^{*}\right)_{k}$ converges in $A P\left(\mathbb{R}, L^{1}\right.$ $([0,1), U))$, it is clear that $\left(\mathbf{S}_{\sigma_{k}} v_{\mathrm{e}}^{*}\right)_{k}$ is a Cauchy sequence in $S^{2}(\mathbb{R}, U)$. Consequently, there exists $N \in \mathbb{N}$ such that $\tilde{\Gamma}_{2}\left\|\mathbf{S}_{\sigma_{k}} v_{\mathrm{e}}^{*}-\mathbf{S}_{\sigma_{\ell}} v_{\mathrm{e}}^{*}\right\|_{S^{2}} \leq \varepsilon / 2$ for all $k, \ell \geq N$. Choosing $\tau \geq 0$ such that $\tilde{\Gamma}_{2} r e^{-\tilde{\gamma} \tau} \leq \varepsilon / 2$, it follows from (4.18) that

$$
\left\|\left(\mathbf{S}_{\sigma_{k}} x_{\mathrm{e}}^{*}\right)(t)-\left(\mathbf{S}_{\sigma_{\ell}} x_{\mathrm{e}}^{*}\right)(t)\right\| \leq \varepsilon \quad \forall t \geq \tau, \forall k, \ell \geq N
$$

The function $\mathbf{S}_{\sigma_{k}} x_{\mathrm{e}}^{*}-\mathbf{S}_{\sigma_{\ell}} x_{\mathrm{e}}^{*}$ is in $A P(\mathbb{R}, X)$, and thus, invoking Lemma 4.1,

$$
\left\|\mathbf{S}_{\sigma_{k}} x_{\mathrm{e}}^{*}-\mathbf{S}_{\sigma_{\ell}} x_{\mathrm{e}}^{*}\right\|_{\infty} \leq \varepsilon \quad \forall k, \ell \geq N
$$

This shows that $\left(\mathbf{S}_{\sigma_{k}} x_{\mathrm{e}}^{*}\right)_{k}$ is a Cauchy sequence in $A P(\mathbb{R}, X)$ and thus converges in $A P(\mathbb{R}, X)$.

Finally, since $\left(\mathbf{S}_{\sigma_{k}} x_{\mathrm{e}}^{*}\right)_{k}$ and $\left(\mathbf{S}_{\sigma_{k}} v_{\mathrm{e}}^{*}\right)_{k}$ are Cauchy sequences in $A P(\mathbb{R}, X)$ and $S^{2}(\mathbb{R}, U)$, respectively, it follows from (4.19) that $\left(\mathbf{S}_{\sigma_{k}} y_{\mathrm{e}}^{*}\right)_{k}$ is a Cauchy sequences in $S^{2}(\mathbb{R}, Y)$, and hence $\left(\mathbf{S}_{\sigma_{k}} \widetilde{y}_{\mathrm{e}}^{*}\right)_{k}$ converges in $A P\left(\mathbb{R}, L^{1}([0,1], Y)\right)$, completing the proof.

We continue by stating a circle-criterion version of Theorem 4.5. 
Theorem 4.7 Let $\Sigma=(\mathbb{T}, \Phi, \Psi, \mathbb{G})$ be a well-posed linear system, let $i, j \in$ $\{1,2\}, K_{1}, K_{2} \in \mathcal{L}\left(Y^{j}, U^{i}\right)$ and let $v^{*} \in S^{2}\left(\mathbb{R}_{+}, U\right)$. Assume that $\Sigma^{j i}=$ $\left(\mathbb{T}, \Phi E^{i}, P^{j} \Psi, P^{j} \mathbb{G} E^{i}\right)$ is optimizable and estimatable and $K_{1} \in \mathbb{S}\left(P^{j} \mathbf{G} E^{i}\right)$ is an admissible feedback operator for $\Sigma^{j i}$. If $\left(I-K_{2} P^{j} \mathbf{G} E^{i}\right)\left(I-K_{1} P^{j} \mathbf{G} E^{i}\right)^{-1}$ is positive real and there exists $\varepsilon>0$ such that $f: Y^{j} \rightarrow U^{i}$ satisfies

$$
\begin{aligned}
& \operatorname{Re}\left\langle f\left(z_{1}\right)-f\left(z_{2}\right)-K_{1}\left(z_{1}-z_{2}\right), f\left(z_{1}\right)-f\left(z_{2}\right)-K_{2}\left(z_{1}-z_{2}\right)\right\rangle \\
& \quad \leq-\varepsilon\left\|z_{1}-z_{2}\right\|^{2} \quad \forall\left(z_{1}, z_{2}\right) \in Y^{j} \times Y^{j},
\end{aligned}
$$

then there exists a unique pair $\left(x^{*}, y^{*}\right) \in A P\left(\mathbb{R}_{+}, X\right) \times S^{2}\left(\mathbb{R}_{+}, Y\right)$ such that $\left(v^{*}, x^{*}, y^{*}\right) \in \mathcal{B}$, for every $\varepsilon>0$, there exists $\delta>0$ such that $P_{2}\left(v^{*}, \delta\right) \subset$ $P\left(x^{*}, \varepsilon\right) \cap P_{2}\left(y^{*}, \varepsilon\right)$ and statements (1)-(5) of Theorem 4.5 hold.

Proof The assumptions are identical to those of Corollary 3.6 with $Z_{1}=Y^{j}$. Consequently, the conclusions of Corollary 3.6 are valid, that is, statements (1)-(4) of Theorem 3.4 hold. Now Theorem 3.4 in turn was the key tool in the proof of Theorem 4.5, and the conclusions of Theorem 4.7 can now be derived by identical arguments provided that, for a given $v^{*} \in S^{2}\left(\mathbb{R}_{+}, U\right)$, there exists a pair $(x, y) \in C\left(\mathbb{R}_{+}, X\right) \times L_{\text {loc }}^{2}\left(\mathbb{R}_{+}, Y\right)$ such that $\left(v^{*}, x, y\right) \in \mathcal{B}$. The existence of such a pair $(x, y)$ can be shown by combining the methods used in the proof of [16, Corollary 4.5] with Lemma 3.2 and Proposition 3.1. We omit the details for the sake of brevity.

We conclude this section with a brief comparison of Theorems 4.5 and 4.7 to related results in the literature. As for the case of periodic forcing, in the finite-dimensional setting, the most relevant results in this context are [31, Theorem 4] and the first part of [48, Theorem 1], both of which are special cases of [16, Corollary 5.6] (which in turn is essentially identical to statement (3) of Theorem 4.5) and [27, Theorem 4.4].

Earlier contributions to the analysis of the asymptotic behaviour of Lur'e systems with almost periodic forcing can be found in $[6,33,34,48]$. The papers $[6,33,34]$ adopt an input-output approach, whilst a standard finite-dimensional state space setting is used in [48]. All of these contributions consider input signals which are almost periodic in the sense of Bohr, but do not cover the more general case of Stepanov almost periodic forcing functions. The structure of the feedback systems and the classes of underlying linear systems considered in $[6,33,34,48]$ are considerably less general than those studied in this paper (in particular, $[6,34,48]$ are restricted to the singleinput single-output case, that is $U$ and $Y$ are one-dimensional and $f$ is a "scalar" nonlinearity). Theorems 4.5 and 4.7 can be considered as far reaching generalizations and refinements of the relevant results in $[6,33,34,48]$.

\section{An example}

We illustrate our results by applying them to a delayed nonlinear mass-spring equation with forcing, namely

$$
m \ddot{z}(t)+d \dot{z}(t-1)+k z(t)+f(z(t-\theta))=v(t),
$$


where the nonlinearity $f: \mathbb{R} \rightarrow \mathbb{R}$ is locally Lipschitz, $f(0)=0$ and the forcing $v \in L_{\text {loc }}^{\infty}\left(\mathbb{R}_{+}\right)$. Here $m, k, d>0$ are constants, $\theta>0$ is a delay, and $z(t)$ denotes the displacement of a mass from rest at time $t$. The damping is linear, but delayed, and the restoring force depends linearly on $z(t)$ and nonlinearly on $z(t-\theta)$.

Letting $\xi(t) \in \mathbb{R}^{2}$ denote the vector in $\mathbb{R}^{2}$ with components $z(t)$ and $\dot{z}(t)$, in firstorder form, (5.1) becomes

$$
\dot{\xi}(t)=A_{0} \xi(t)+A_{1} \xi(t-1)+B_{0} f\left(C_{0} \xi(t-\theta)\right)-B_{0} v(t),
$$

where

$$
A_{0}:=\left(\begin{array}{cc}
0 & 1 \\
-k / m & 0
\end{array}\right), \quad A_{1}:=\left(\begin{array}{cc}
0 & 0 \\
0 & -d / m
\end{array}\right), \quad B_{0}:=\left(\begin{array}{c}
0 \\
-1 / m
\end{array}\right) \quad \text { and } \quad C_{0}:=(1,0)
$$

Setting $h:=\max \{\theta, 1\}$, system (5.2) may be written in the form (3.1) on the statespace $M^{2}:=M^{2}\left([-h, 0], \mathbb{R}^{2}\right)=\mathbb{R}^{2} \times L^{2}\left([-h, 0], \mathbb{R}^{2}\right)$ (see, for example [11, Sect. 2.4]). To this end, define

$$
A\left(w_{0}, w_{1}\right):=\left(A_{0} w_{0}+A_{1} w_{1}(-1), \dot{w}_{1}\right) \quad \forall w=\left(w_{0}, w_{1}\right) \in D(A),
$$

and

$$
D(A):=\left\{\left(w_{0}, w_{1}\right) \in M^{2}: w_{1} \in W^{1,2}\left([-h, 0], \mathbb{R}^{2}\right) \text { and } w_{0}=w_{1}(0)\right\} .
$$

Further, we set

$$
\begin{aligned}
& B r:=\left(B_{0} r, 0\right) \in M^{2} \quad \forall r \in \mathbb{R}, \quad B_{\mathrm{e}}:=-B, \\
& C w:=C_{0} w_{1}(-\theta) \quad \forall w=\left(w_{0}, w_{1}\right) \in D(A) .
\end{aligned}
$$

It is well known that $\left(A,\left(B, B_{\mathrm{e}}\right), C\right)$ generates a (regular) well-posed system with transfer function given by $C(s I-A)^{-1}\left(B, B_{\mathrm{e}}\right)=(\mathbf{G}(s),-\mathbf{G}(s))$, where

$$
\mathbf{G}(s)=C(s I-A)^{-1} B=-\frac{e^{-s \theta}}{m s^{2}+d s e^{-s}+k} .
$$

Setting $x(t)=\left(\xi(t), \xi_{t}\right)$ with $\xi_{t}(s):=\xi(t+s)$ for $s \in[-h, 0]$, the delay differential system (5.2) can be written as

$$
\dot{x}=A x+B f(C x)+B_{\mathrm{e}} v=A x+\left(B, B_{\mathrm{e}}\right)(f(C x), v)^{T}, \quad x(0)=x^{0} .
$$

By invoking the operator families generated by $A,\left(B, B_{\mathrm{e}}\right)$ and $C$, the system (5.3) may be reformulated in terms of operator families and embedded into the four-block form (3.1) by choosing $U^{1}=U^{2}=Y^{1}=\mathbb{R}$ and $Y^{2}=\{0\}$. For brevity, we do not give the details. Noting that

$$
\mathbf{G}(s) \rightarrow 0 \text { as } \operatorname{Re}(s) \rightarrow \infty
$$


it follows from Proposition 3.1 that, for each globally Lipschitz $f$, all $x^{0} \in M^{2}$ and $v \in L_{\text {loc }}^{2}\left(\mathbb{R}_{+}\right)$, the Lur'e system (5.3) has a unique solution defined on $\mathbb{R}_{+}$.

The spectrum of $A$ consists of the zeros of the quasi-polynomial $P(s):=m s^{2}+$ $d s e^{-s}+k$, and it follows from [41, Theorem 3.8] that $P$ does not have any zeros in the closed right-half plane if, and only if,

$$
k>0 \text { and } 0<\frac{d}{m}<\frac{\pi}{2}-\frac{2 k}{\pi m} .
$$

As the strongly continuous semigroup generated by $A$ has the spectrum determined growth property, the exponential stability of this semigroup is guaranteed if (5.4) holds.

Common to all following simulations, we take

$$
m=k=1, \quad d=0.5 \text { and } \theta=2,
$$

and so (5.4) is satisfied and $h=\theta=2$. We compute numerically that $\|\mathbf{G}\|_{H^{\infty}} \approx$ $5.43=: g$. Furthermore, with $\zeta \in L^{2}([-h, 0])$ defined by

$$
\zeta(t):= \begin{cases}-1 & t \in[-h,-h / 2) \\ 0 & t=-h / 2 \\ 1 & t \in(-h / 2,0]\end{cases}
$$

we choose the initial condition

$$
x^{0}=\left((\zeta(0),-\zeta(0))^{T},(\zeta,-\zeta)^{T}\right)
$$

As nonlinearity $f$ we choose the saturating function

$$
f(y)=(0.95 / g)\left(\frac{y}{1+|y|}\right) \forall y \in \mathbb{R}
$$

(see Fig. 3a for an illustration of the graph of $f$ ). The function $f$ is globally Lipschitz with Lipschitz constant $0.95 / g<1 / g=1 /\|\mathbf{G}\|_{H^{\infty}}$. Therefore, $f$ satisfies the condition (3.2) with $Z_{1}=Z_{2}=\mathbb{R}$ and $K^{i j}=0$.

To summarize, with the model data (5.5) and (5.6), the Lur'e system (5.3) satisfies the hypotheses of Theorems 3.4 and 4.5 . For a numerical illustration of Theorem 4.5, we consider the following inputs defined on $\mathbb{R}_{+}$

$$
\begin{aligned}
v^{\mathrm{p}}(t) & =\sin \left(2 \pi \omega_{1} t\right), & v^{\mathrm{ap}}(t) & =\sin \left(2 \pi \omega_{1} t\right)+\sin \left(2 \pi \omega_{2} t\right), \\
v^{\mathrm{aap}}(t) & =v^{\mathrm{ap}}(t)+2 /\left(1+\pi\left(\omega_{1}+\omega_{2}\right) t^{2}\right), & & v^{\mathrm{s}}(t)=\omega_{1} \bmod \left(t, 1 / \omega_{1}\right)+\omega_{2} \bmod \left(t, 1 / \omega_{2}\right), \\
v^{\mathrm{as}}(t) & =\left(1+t e^{-0.9 t}\right) v^{\mathrm{s}}(t), & &
\end{aligned}
$$




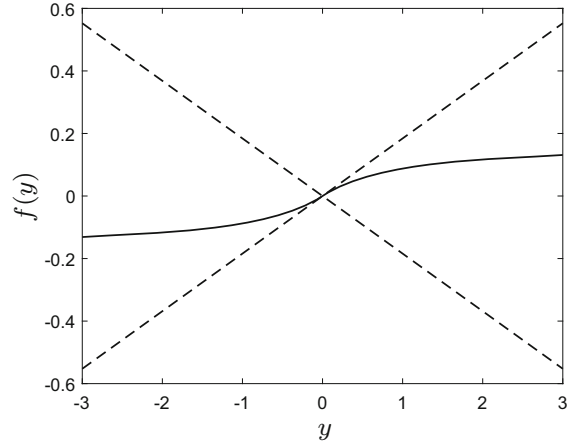

(a)

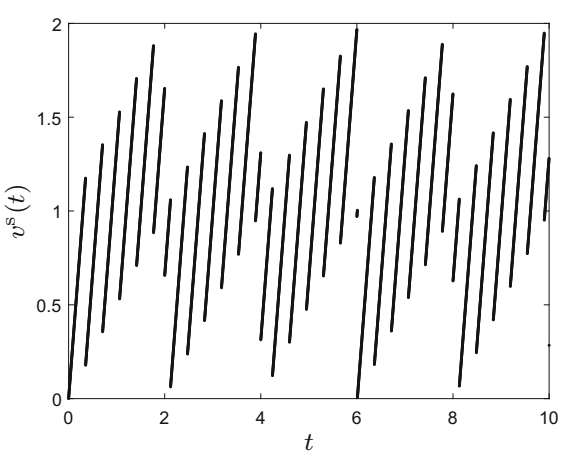

(b)

Fig. 3 a Graph of $f$ (solid) and straight lines with slopes $\pm g$ (dashed). b Graph of $v^{\mathrm{s}}$

with $\omega_{1}=0.5$ and $\omega_{2}=2 \sqrt{2}$. Here, for fixed $\tau>0$, the function $\bmod (\cdot, \tau): \mathbb{R}_{+} \rightarrow$ $[0, \tau)$ is defined by

$\bmod (t, \tau)=t-k \tau \in[0, \tau), \quad$ where $k$ is the largest integer in $\mathbb{Z}_{+}$such that $t \geq k \tau$.

The functions $v^{\mathrm{p}}, v^{\mathrm{ap}}, v^{\mathrm{aap}}, v^{\mathrm{s}}$, $v^{\text {as }}$ are, respectively, periodic, almost periodic (in the sense of Bohr), asymptotically almost periodic, Stepanov almost periodic and asymptotically Stepanov almost periodic. The graph of $v^{\mathrm{s}}$ is plotted in Fig. 3b.

We note that $v^{\text {ap }}$ is the sum of two periodic functions, and hence almost periodic. However, as the periods of the two summands are not commensurate, $v^{\text {ap }}$ is not periodic (as follows from [26, Theorem 2.1] or by a routine contradiction argument). Furthermore, for each $\tau>0$, the function $\bmod (\cdot, \varnothing) \in L_{\text {loc }}^{2}\left(\mathbb{R}_{+}\right)$is periodic, but not continuous. Note that $v^{\mathrm{s}}$ is the linear combination of two mod functions with incommensurable periods, and so is not periodic by [26, Theorem 2.2]. (Again this can be shown directly by an elementary argument.) Each of the summands on the right-hand side of the defining equation for $v^{\mathrm{s}}$ is periodic, and hence so are their Bochner transforms. Thus, the sum of Bochner transforms is almost periodic (in the sense of Bohr), and hence $v^{\mathrm{s}}$ is almost periodic in the sense of Stepanov, that is, $v^{\mathrm{s}} \in S^{2}\left(\mathbb{R}_{+}\right)$.

Simulation results are contained in Figs. 4-6. The numerical solutions of (5.3) were computed using MATLAB's dde23 delay differential equation solver. For $\zeta$ and $x^{0}$ as above, and for $v \in L_{\text {loc }}^{\infty}\left(\mathbb{R}_{+}\right)$, we denote the solution of $(5.3)$ by $x\left(\cdot ; x^{0}, v\right)$, and, for notational convenience, we write $x\left(t ; x^{0}, v\right)=\left(\xi(t ; \zeta, v), \xi_{t}(\cdot ; \zeta, v)\right)$ and

$$
\xi(t ; \zeta, v)=\left(\begin{array}{l}
z(t ; \zeta, v) \\
\dot{z}(t ; \zeta, v)
\end{array}\right)=\left(\begin{array}{l}
z(t) \\
\dot{z}(t)
\end{array}\right)
$$

In Fig. 4 we see that $\xi\left(t ; \zeta, v^{\mathrm{p}}\right)$ converges to a periodic function over time, guaranteed theoretically by statement (3) of Theorem 4.5.

Panels (a) and (b) of Fig. 5 show the first and second components of $\xi\left(t ; \zeta, v^{\text {ap }}\right)$ (solid) and $\xi\left(t ; \zeta, v^{\text {aap }}\right)($ dashed $)$, respectively. Convergence $\left\|\xi\left(t ; \zeta, v^{\text {ap }}\right)-\xi\left(t ; \zeta, v^{\text {aap }}\right)\right\|$ 


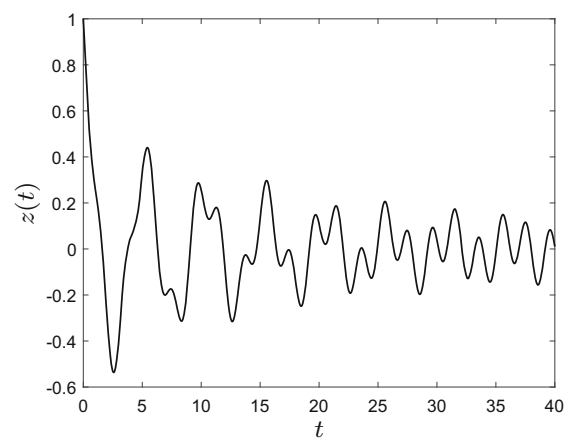

(a)

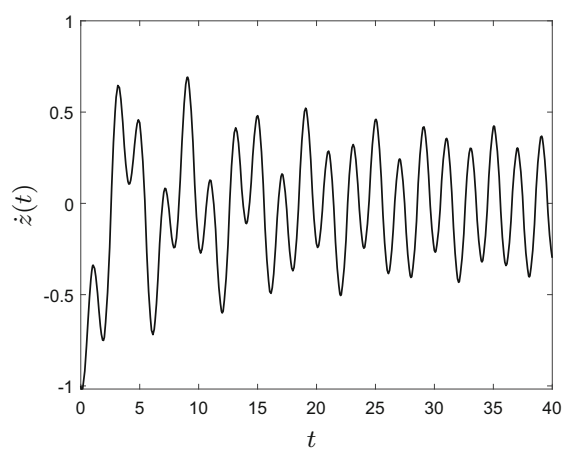

(b)

Fig. 4 a Graph of $z\left(t ; \xi, v^{\mathrm{p}}\right)$. b Graph of $\dot{z}\left(t ; \xi, v^{\mathrm{p}}\right)$

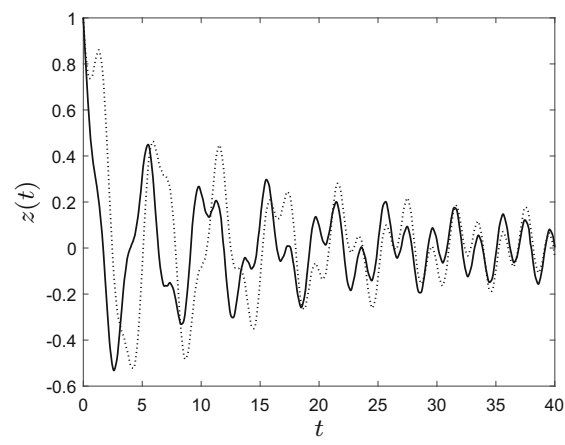

(a)

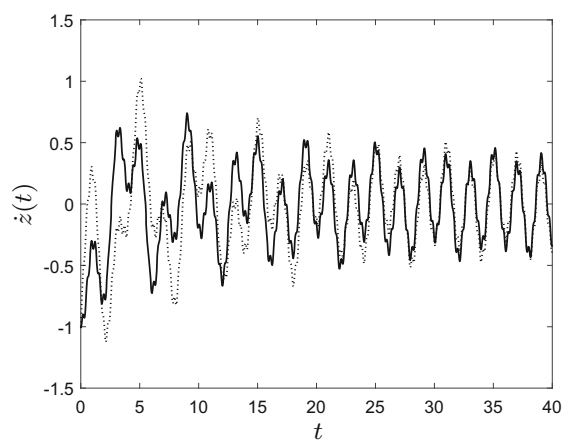

(b)

Fig. 5 a $z\left(t ; \xi, v^{\text {ap }}\right)$ (solid line) and $z\left(t ; \xi, v^{\text {aap }}\right)$ (dotted line). $\mathbf{b} \dot{z}\left(t ; \xi, v^{\text {ap }}\right)$ (solid line) and $\dot{z}\left(t ; \xi, v^{\text {aap }}\right)$ (dotted line)

$\rightarrow 0$ is seen as $t \rightarrow \infty$, and $\xi\left(t ; \zeta, v^{\text {ap }}\right)$ converges to an almost periodic function as $t \rightarrow \infty$, as is guaranteed by statement (1) of Theorem 4.5 .

Figure 6 shows, in panels (a) and (b), the first and second components of $\xi\left(t ; \zeta, v^{\mathrm{s}}\right)$ (solid) and $\xi\left(t ; \zeta, v^{\mathrm{as}}\right)$ (dashed), respectively. Convergence $\| \xi\left(t ; \zeta, v^{\mathrm{s}}\right)-$ $\xi\left(t ; \zeta, v^{\text {as }}\right) \| \rightarrow 0$ as $t \rightarrow \infty$, guaranteed by statement (3) of Theorem 4.5 , is illustrated by the simulation. Furthermore, the simulation also shows convergence of $\xi\left(t ; \zeta, v^{\mathrm{s}}\right)$ to an almost periodic function as $t \rightarrow \infty$, although the exact (almost) periodic nature of the limiting function is arguably unclear to the eye.

\section{Conclusions}

The analysis of solutions of differential equations, the right-hand side of which exhibits almost periodic time dependence, has a long history. In the context of a general class of forced infinite-dimensional Lur'e systems, we have addressed typical questions such as 


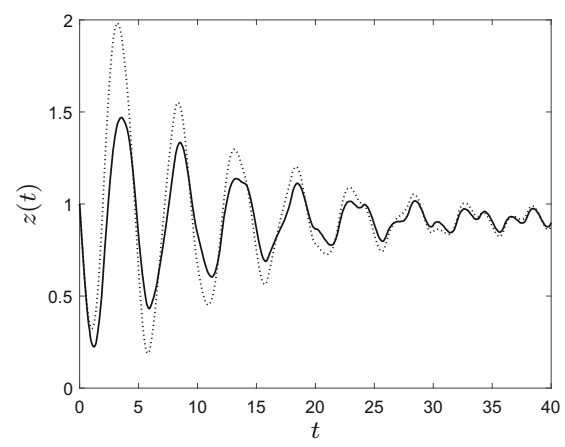

(a)

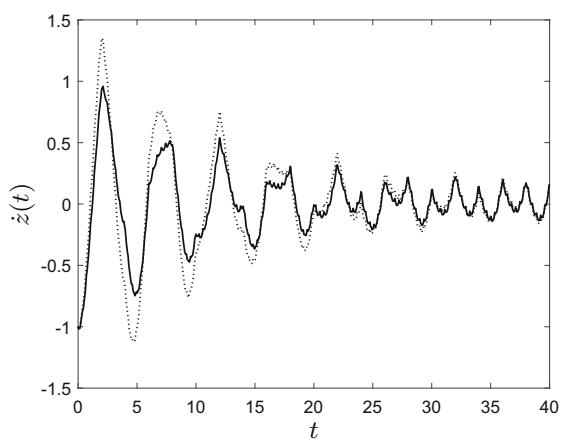

(b)

Fig. $6 \mathbf{a} z\left(t ; \xi, v^{\mathrm{s}}\right)$ (solid line) and $z\left(t ; \xi, v^{\text {as }}\right)$ (dotted line). $\mathbf{b} \dot{z}\left(t ; \xi, v^{\mathrm{s}}\right)$ (solid line) and $\dot{z}\left(t ; \xi, v^{\text {as }}\right)$ (dotted line)

- Does there exist a globally attracting almost periodic solution corresponding to a given (asymptotically) almost periodic forcing function?

- Are bounded bilateral solutions almost periodic?

By using ideas from ISS and classical absolute stability theory, we have developed natural criteria which provide sufficient conditions under which the answer to the above questions is positive. More specifically, we have analysed the asymptotic behaviour of infinite-dimensional Lur'e systems with Stepanov almost periodic forcing. The linear dynamic component of the Lur'e system is a well-posed infinite-dimensional systems without any smoothing conditions imposed. Hence, the output is potentially highly irregular and therefore cannot be (asymptotically) almost periodic in the sense of Bohr in general. Consequently, Stepanov almost periodicity is the natural concept of almost periodicity for the class of Lur'e systems under consideration. Under conditions reminiscent of those featuring in the complex Aizerman conjecture and the circle criterion, our criteria guarantee that every state/output pair $(x, y)$ generated by a given asymptotically Stepanov almost periodic input signal $v$ is asymptotically almost periodic ( $x$ in the sense of Bohr and $y$ in the sense of Stepanov), the almost periodic parts $x^{*}$ and $y^{*}$ of $x$ and $y$, respectively, are completely determined by the almost periodic part $v^{*}$ of $v,\left(v^{*}, x^{*}, y^{*}\right)$ is a trajectory, and the $\varepsilon$-periods of $x^{*}$ and $y^{*}$ contain the $\delta$-periods of $v$, provided that $\delta>0$ is sufficiently small. The almost periodic bilateral extension $\left(v_{\mathrm{e}}^{*}, x_{\mathrm{e}}^{*}, y_{\mathrm{e}}^{*}\right)$ of $\left(v^{*}, x^{*}, y^{*}\right)$ is a bilateral trajectory and the bilateral state/output pair $\left(x_{\mathrm{e}}^{*}, y_{\mathrm{e}}^{*}\right)$ is unique in the sense that there is no other bilateral state/output pair $(\hat{x}, \hat{y})$ such that $\hat{x}$ is bounded and $\left(v_{\mathrm{e}}^{*}, \hat{x}, \hat{y}\right)$ is a bilateral trajectory.

Open Access This article is licensed under a Creative Commons Attribution 4.0 International License, which permits use, sharing, adaptation, distribution and reproduction in any medium or format, as long as you give appropriate credit to the original author(s) and the source, provide a link to the Creative Commons licence, and indicate if changes were made. The images or other third party material in this article are included in the article's Creative Commons licence, unless indicated otherwise in a credit line to the material. If material is not included in the article's Creative Commons licence and your intended use is not permitted by statutory regulation or exceeds the permitted use, you will need to obtain permission directly from the copyright holder. To view a copy of this licence, visit http://creativecommons.org/licenses/by/4.0/. 


\section{References}

1. Amerio L, Prouse G (1971) Almost periodic functions and functional equations. Springer, New York

2. Angeli D (2002) A Lyapunov stability approach to incremental stability properties. IEEE Trans Autom Control 37:410-421

3. Arcak M, Teel A (2002) Input-to-state stability for a class of Lurie systems. Automatica 38:1945-1949

4. Bart H, Goldberg S (1978) Characterizations of almost periodic strongly continuous groups and semigroups. Math Ann 236:105-116

5. Bellow A, Losert V (1985) The weighted pointwise ergodic theorem and the individual ergodic theorem along subsequences. Trans Am Math Soc 288:307-345

6. Běnes VE, Sandberg IW (1965) On the response of time-variable nonlinear systems to almost periodic signals. J Math Anal Appl 10:245-268

7. Bohr H (1947) Almost periodic functions. Chelsea Publishing Company, New York

8. Corduneanu C (1989) Almost periodic functions, 2nd edn. Chelsea Publishing Company, New York

9. Corduneanu C (2009) Almost periodic oscillations and waves. Springer, New York

10. Curtain RF, Logemann H, Staffans O (2003) Stability results of Popov-type for infinite-dimensional systems with applications to integral control. Proc Lond Math Soc 86:779-816

11. Curtain RF, Zwart H (1995) An introduction to infinite-dimensional linear systems theory. Springer, New York

12. Dashkovskiy SN, Efimov DV, Sontag ED (2011) Input-to-state stability and allied system properties. Autom Remote Control 72:1579-1614

13. Desoer CA, Vidyasagar M (1975) Feedback systems: input-output properties. Academic Press, New York

14. Fink AM (1974) Almost periodic differential equations. Springer, Berlin

15. Guiver C, Logemann H, Opmeer MR (2017) Transfer functions of infinite-dimensional systems: positive realness and stabilization. Math Control Signals Syst. https://doi.org/10.1007/s00498-0170203-z

16. Guiver C, Logemann H, Opmeer MR (2019) Infinite-dimensional Lur'e systems: input-to-state stability and convergence properties. SIAM J Control Optim 57:334-365

17. Guiver C, Logemann H, Townley S (2017) Low-gain integral control for multi-input multi-output linear systems with input nonlinearities. IEEE Trans Automat Control 62:4776-4783

18. Haddad WM, Chellaboina V (2008) Nonlinear dynamical systems and control. Princeton University Press, Princeton

19. Hinrichsen D, Pritchard AJ (1995) Destabilization by output feedback. Differ Integral Eqn 5:357-386

20. Hinrichsen D, Pritchard AJ (2005) Mathematical systems theory i. Springer, Berlin

21. Jayawardhana B, Logemann H, Ryan EP (2009) Input-to-state stability of differential inclusions with applications to hysteretic and quantized feedback systems. SIAM J Control Optim 48:1031-1

22. Jayawardhana B, Logemann H, Ryan EP (2011) The circle criterion and input-to-state stability: new perspectives on a classical result. IEEE Control Syst Magn 31:32-67

23. Karafyllis I, Krstic M (2019) Input-to-state stability for PDEs. Springer, Cham

24. Mikkola KM (2006) State-feedback stabilization of well-posed linear systems. Integral Equ Oper Theory 55:249-271

25. Mironchenko A, Prieur C (2019) Input-to-state stability of infinite-dimensional systems: recent results and open questions, arXiv preprint. arXiv:1910.01714

26. Mirotin AR, Mirotin EA (2009) On sums and products of periodic functions. Real Anal Exchange 34:347-358

27. Natarajan V, Weiss G (2013) Behavior of a stable nonlinear infinite-dimensional system under the influence of a nonlinear exosystem. 1st IFAC workshop on control of systems governed by PDEs, Paris $155-160$

28. Ruess WM, Summers WH (1988) Compactness in spaces of vector-valued continuous functions and asymptotic almost periodicity. Math Nachr 135:7-33

29. Salamon D (1987) Infinite-dimensional linear systems with unbounded control and observation: a functional analytic approach. Trans Am Math Soc 300:383-431

30. Salamon D (1989) Realization theory in Hilbert space. Math Syst Theory 21:147-164

31. Sandberg IW (1965) Some results on the theory of physical systems governed by nonlinear functional equations. Bell Syst Tech J 44:871-898 
32. Sandberg IW (1981) Criteria for the response of nonlinear systems to be $L$-asymptotically periodic. Bell Syst Tech J 60:2359-2371

33. Sandberg IW (2000) The circle criterion and almost periodic inputs. IEEE Trans Circuits Syst I Fund Theory Appl 47:825-829

34. Sandberg IW, van Zyl GJJ (2001) The spectral coefficients of the response of nonlinear systems to asymptotically almost periodic inputs. IEEE Trans Circuits Syst I Fund Theory Appl 48:170-176

35. Sarkans E, Logemann H (2015) Input-to-state stability of Lur'e systems. Math Control Signals Syst 27:439-465

36. Schwenninger F (2019) Input-to-state stability for parabolic boundary control: linear and semi-linear systems, arXiv preprint. arXiv:1908.08317

37. Sontag ED (1989) Smooth stabilization implies coprime factorization. IEEE Trans Autom Control $34: 435-443$

38. Sontag ED (2006) Input to state stability: basic concepts and results. In: Nistri P, Stefani G (eds) Nonlinear and optimal control theory. Springer, Berlin, pp 163-220

39. Staffans OJ (2005) Well-posed linear systems. Cambridge University Press, Cambridge

40. Staffans OJ, Weiss G (2002) Transfer functions of regular linear systems. Part II: the systems operator and the Lax-Phillips semigroup. Trans Am Math Soc 354:3229-3262

41. Stépán G (1989) Retarded dynamical systems: stability and characteristic functions. Longman Scientific \& Technical, Madison

42. Tucsnak M, Weiss G (2009) Observation and control for operator semigroups. Birkhäuser, Berlin

43. Tucsnak M, Weiss G (2014) Well-posed systems - the LTI case and beyond. Automatica 50:1757-1779

44. Vidyasagar M (1993) Nonlinear systems analysis, 2nd edn. Prentice-Hall, Englewood Cliffs

45. Weiss G (1994) Transfer functions of regular linear systems. Part I: characterizations of regularity Trans. Am Math Soc 342:827-854

46. Weiss G (1994) Regular linear systems with feedback. Math Control Signals Syst 7:23-57

47. Weiss G, Rebarber R (2000) Optimizability and estimatability for infinite-dimensional linear systems. SIAM J Control Optim 39:1204-1232

48. Yakubovich VA (1964) Matrix inequalities method in stability theory for nonlinear control systems: I. Absolute stability of forced vibrations. Autom Remote Control 7:905-917

49. Yakubovich VA, Leonov GA, Gelig AKh (2004) Stability of stationary sets in control systems with discontinuous nonlinearities. World Scientific, Singapore

Publisher's Note Springer Nature remains neutral with regard to jurisdictional claims in published maps and institutional affiliations. 